\title{
Formation, Orbital and Internal Evolutions of Young Planetary Systems
}

\author{
Clément Baruteau ${ }^{1} \cdot$ Xuening Bai $^{2}$. \\ Christoph Mordasini ${ }^{3}$ Paul Mollière ${ }^{4}$
}

Received: 22 September 2015 / Accepted: 27 April 2016 / Published online: 12 May 2016

(C) Springer Science+Business Media Dordrecht 2016

\begin{abstract}
The growing body of observational data on extrasolar planets and protoplanetary disks has stimulated intense research on planet formation and evolution in the past few years. The extremely diverse, sometimes unexpected physical and orbital characteristics of exoplanets lead to frequent updates on the mainstream scenarios for planet formation and evolution, but also to the exploration of alternative avenues. The aim of this review is to bring together classical pictures and new ideas on the formation, orbital and internal evolutions of planets, highlighting the key role of the protoplanetary disk in the various parts of the theory. We begin by briefly reviewing the conventional mechanism of core accretion by the growth of planetesimals, and discuss a relatively recent model of core growth through the accretion of pebbles. We review the basic physics of planet-disk interactions, recent progress in this area, and discuss their role in observed planetary systems. We address the most important effects of planets internal evolution, like cooling and contraction, the massluminosity relation, and the bulk composition expressed in the mass-radius and mass-mean density relations.
\end{abstract}

Keywords Planets and satellites: formation · Planets and satellites: interiors ·

Protoplanetary disks $\cdot$ Planet-disk interactions

\section{Introduction}

Planet formation and evolution is a fast-moving field, stimulated by the rapid increase in the number of exoplanets and their great diversity. Despite the wealth of observational

\footnotetext{
C. Baruteau

clement.baruteau@irap.omp.eu

1 Institut de Recherche en Astrophysique et Planétologie, CNRS/Université de Toulouse, Toulouse, France

2 Harvard-Smithsonian Center for Astrophysics, Cambridge, USA

3 Physikalisches Institut, Universität Bern, Bern, Switzerland

4 Max-Planck-Institut für Astronomie, Heidelberg, Germany
} 
data on planetary systems, including our own, it is difficult to have a general theory for planet formation and evolution as it involves a broad range of physical processes that happen at widely different length and time scales. The protoplanetary disk where planets form embodies many of the difficulties and uncertainties related to planet formation and evolution. Protoplanetary disks are made of dust and poorly-ionized gas governed by non-ideal magneto-hydrodynamics. The disk's structural and turbulent properties are thus influenced by complex radiative and chemical processes, which set the thermodynamical behavior and ionization state of the gas. They have critical implications in every part of planet formation and evolution: (i) they condition the location in protoplanetary disks where dust grains grow to planetesimals and to planets, (ii) they play a prominent role in the early orbital evolution of low-mass planetary systems by changing the direction and speed of planetary migration, (iii) they impact the early internal evolution of planets by determining the amount of heat retained from the formation process. The formation and evolution of planetary systems is obviously affected by gravity and by the local stellar environment. This broad range of physical processes echoes the diversity of extrasolar planetary systems, and suggests that there is likely no unique theory for planet formation and evolution, but several.

Dramatic progress in understanding planet formation and evolution in the past few years has motivated a number of recent reviews (e.g., Raymond et al. 2014; Helled et al. 2014; Baruteau et al. 2014; Chabrier et al. 2014; Baraffe et al. 2014). The aim of this chapter is to provide an update. It brings together the formation, the orbital and internal evolutions of young planetary systems as all three are intimately linked. For instance, the radius and luminosity of a young planet depends on its formation scenario, and so does its orbital migration. The energy released by a forming planet as it cools and contracts can change the properties of the protoplanetary disk in the planet's vicinity and thus affect its orbital migration as much as its growth. This chapter thus puts an accent on, but is not restricted to, the early stages of planetary formation and evolution, before the dispersal of the protoplanetary disk. It begins with a review on the growth of planetary cores by planetesimal and pebble accretions in Sect. 2. It continues with the orbital evolution of planets driven by planet-disk interactions, which is presented in Sect. 3. The internal evolution of planets follows in Sect. 4. Summary points are provided in Sect. 5 .

\section{Growth of Planetesimals to Protoplanets}

The journey of planet formation begins with the growth of dust grains in protoplanetary disks and the formation of planetesimals, as reviewed in the previous chapter by Birnstiel et al. Due to major uncertainties in our understanding of planetesimal formation, most studies of the later phase of planet formation assume a population of planetesimals are readily in place to start with, and follow the growth of planetesimals into planetary mass objects, or protoplanets. If the protoplanets reach sufficiently high mass within the lifetime of their parent protoplanetary disk, they can further accrete gas and become the cores of giant planets (i.e., the "core accretion" paradigm for giant planet formation). For this reason, the terms "protoplanet" and "core" are sometimes used interchangeably in the literature.

\subsection{Planetesimal Accretion}

We begin by briefly reviewing the conventional mechanism of planetesimal growth, where planetesimals grow by accreting other planetesimals. The theory has been developed and refined over decades. Given the limited space of this chapter and the maturity of this subject, 
we simply introduce the fundamental concepts of planetesimal accretion. A pedagogical introduction can be found in the book by Armitage (2010), and a more in-depth review was given by Goldreich et al. (2004). Applications to terrestrial and extrasolar planetary systems are summarized in the recent PPVI chapters by Raymond et al. (2014) and Helled et al. (2014).

\subsubsection{Gravitational Focusing and Runaway Growth}

Consider a swarm of planetesimals, with surface density $\Sigma_{\mathrm{p}}$, characteristic radial size $R$ (corresponding mass $M$ ), and velocity dispersion $v_{\mathrm{p}}$. They are located around disk radius $a$, where the disk's angular frequency is $\Omega_{\mathrm{K}}$ (equal to the Keplerian angular frequency). These planetesimals are thus distributed over a vertical thickness of $H \approx v_{\mathrm{p}} / \Omega_{\mathrm{K}}$, with corresponding midplane number density $n_{\mathrm{p}} \approx \Sigma_{\mathrm{p}} / M H \approx \Sigma_{\mathrm{p}} \Omega_{\mathrm{K}} / M v_{\mathrm{p}}$.

Assuming every collision among the planetesimals leads to coagulation, the rate of planetesimal growth is given by, within order unity,

$$
\frac{d M}{d t} \approx n_{\mathrm{p}} M \sigma v_{\mathrm{rel}} \approx \Sigma_{\mathrm{p}} \Omega_{\mathrm{K}} \sigma
$$

where $v_{\text {rel }} \approx \sqrt{2} v_{\mathrm{p}}$ is the typical relative velocity of planetesimal encounters, and the corresponding collisional cross section $\sigma$ is

$$
\sigma=\pi R^{2}\left(1+\frac{v_{\mathrm{esc}}^{2}}{v_{\mathrm{rel}}^{2}}\right),
$$

where $v_{\mathrm{esc}}^{2}=2 G M / R$ is the escape velocity. The second term is called gravitational focusing.

When the planetesimals' mass is small, or when the population of planetesimals are "hot" (with large velocity dispersions $v_{\mathrm{p}}$ ), we have $v_{\text {esc }} \ll v_{\text {rel }}$, and hence the collisional cross section is simply geometric. Correspondingly, $d M / d t \propto R^{2} \propto M^{2 / 3}$, or $d(\ln M) / d t \propto$ $M^{-1 / 3} \propto R^{-1}$. This is called ordered growth. If we rewrite the mass growth rate to growth rate in planetesimal radius, it becomes $d R / d t \approx$ const: planetesimals of all sizes grow at the same rate.

As planetesimals become more massive, or when their relative velocity is sufficiently low so that $v_{\text {esc }} \gtrsim v_{\text {rel }}$, gravitational focusing significantly enhances the cross section. At fixed $v_{\text {rel }}$, gravitational focusing is more effective for more massive planetesimals. This leads to the so-called runaway growth, where we have $d M / d t \propto R^{2} \times(M / R) \propto M^{4 / 3}$, or $d \ln M / d t \propto M^{1 / 3} \propto R$. In this regime, the growth of larger bodies dramatically runaways over smaller bodies, and as a result, few massive bodies stand out over the rest of the planetesimals population, as firstly found from numerical studies (Greenberg et al. 1978; Wetherill and Stewart 1989; Kokubo and Ida 1996).

A key requirement for runaway growth is that the velocity dispersion $v_{\mathrm{p}}$ for the bulk of the (small) planetesimal population is kept small. This is a result of the balance among various dynamical heating and cooling processes. In general, larger bodies resulting from runaway growth are cooled by dynamical friction with small bodies, which contain most of the mass. The small body populations are heated during this process, but they cool by mutual collisions and gas drag. 


\subsubsection{Oligarchic Growth, Isolation Mass and Timescales}

At late stages of the runaway growth, planetary embryos become sufficiently large and start to interact with each other, and the overall dynamics is dominated by these few bodies, called the oligarchs. They tend to heat up the neighboring planetesimals, increasing their velocity dispersion approximately as $v_{\mathrm{p}} \propto M^{1 / 3}$. More precisely, N-body simulations suggest that the root mean square planetesimal eccentricity $e$ and inclination $i$ scale with embryo mass as (e.g., Ida and Makino 1993; Kokubo and Ida 2000)

$$
\left\langle e^{2}\right\rangle^{1 / 2} \sim 6 R_{\mathrm{H}} / a, \quad\left\langle i^{2}\right\rangle^{1 / 2} \sim 3.5 R_{\mathrm{H}} / a,
$$

where

$$
R_{\mathrm{H}}=\left(\frac{G M}{3 \Omega_{\mathrm{K}}^{2}}\right)^{1 / 3}=\left(\frac{M}{3 M_{*}}\right)^{1 / 3} a
$$

is the embryo's Hill radius, and $M_{*}$ is the stellar mass. This enhancement of velocity dispersion reduces the efficiency of gravitational focusing, and hence slows down the growth of planetary embryos, a regime called oligarchic growth (Kokubo and Ida 1998, 2000), with growth rate scaled as $d M / d t \propto R^{2} \times(M / R) / M^{2 / 3} \propto M^{2 / 3}$, or $d \ln M / d t \propto M^{-1 / 3} \propto R^{-1}$. Therefore, the growth mode of the large embryos reduces to orderly. In other words, neighboring oligarchs growth at similar rates and maintain similar masses. On the other hand, the collisional cross section continues to be dominated by gravitational focusing, thus large embryos runaway over small planetesimals. Therefore, the overall outcome of runaway growth followed by oligarchic growth is a bi-modal distribution of an embryo-planetesimal system.

During oligarchic growth, each planetary embryo establishes its own domain of dominance, and maintains certain separation with neighboring embryos. Empirically, it was found that the separation $\Delta a$ is about $10 R_{\mathrm{H}}$ (Kokubo and Ida 1995, 1998). If it were closer, planet embryos would experience close encounters, and such strong scattering would increase their eccentricity and expand their separation. Circularization is then achieved due to dynamical friction from the planetesimals. Therefore, the maximum mass a planet embryo can achieve by planetesimal accretion is limited by the amount of planetesimals available in its feeding zone. This mass is called the isolation mass, given by $M_{\mathrm{iso}} \approx 2 \pi a \Delta a \Sigma_{\mathrm{p}}$. Assuming $\Delta a=10 R_{\mathrm{H}}$ and $M_{*}=M_{\odot}$, this mass is found to be

$$
M_{\text {iso }} \approx 50\left(\frac{\pi a^{2} \Sigma_{\mathrm{p}}}{M_{*}}\right)^{3 / 2} M_{*} \approx 0.16\left(\frac{\Sigma_{\mathrm{p}}}{10 \mathrm{~g} \mathrm{~cm}^{-2}}\right)^{3 / 2}\left(\frac{a}{1 \mathrm{AU}}\right)^{3} M_{\oplus} .
$$

The isolation mass mainly depends on radius and surface density of planetesimals as $\left(\Sigma_{\mathrm{p}} a^{2}\right)^{3 / 2}$. Although the scaling with $a$ is not well known, we generally expect $\Sigma_{\mathrm{p}}$ to decrease with radius slower than $a^{-2}$ (e.g., $\Sigma_{\mathrm{p}} \propto a^{-1.5}$ in the Minimum-Mass Solar Nebula model - hereafter MMSN), and hence $M_{\text {iso }}$ increases with $a$.

We can roughly estimate the growth timescale during the oligarchic growth phase. Using Eqs. (3), we get the typical velocity dispersion $v_{\mathrm{p}} \sim 5 R_{\mathrm{H}} \Omega_{\mathrm{K}}$, and we substitute it into Eqs. (1) and (2) to obtain

$$
\begin{aligned}
\tau_{\text {grow }} \approx \frac{M}{d M / d t} & \approx 8 \Omega_{\mathrm{K}}^{-1}\left(\frac{M_{*}}{\Sigma_{\mathrm{p}} a^{2}}\right)\left(\frac{R_{\mathrm{H}}^{2}}{a R}\right) \\
& \approx 1.2\left(\frac{M}{0.1 M_{\oplus}}\right)^{1 / 3}\left(\frac{\Sigma_{\mathrm{p}}}{10 \mathrm{~g} \mathrm{~cm}^{-2}}\right)^{-1}\left(\frac{a}{1 \mathrm{AU}}\right)^{1 / 2} \text { Myrs. }
\end{aligned}
$$


The results above suggest that Mercury and Mars may be the leftover embryos from oligarchic growth, and they form within a few million years, most likely within the lifetime of the Solar nebular. Faster growth is possible when considering the accretion of small planetesimals where gas drag efficiently damps their eccentricities (Rafikov 2004; Kenyon and Bromley 2009), the overall result is a reduction of the pre-factor in Eq. (6) but not the scaling.

Upon accreting most planetesimals from their feeding zones to achieve the isolation mass, there is insufficient damping of random velocities of the planetary embryos from dynamical friction (Kenyon and Bromley 2006). This marks the end of oligarchic growth, and the system becomes chaotic, with eccentricity growth, orbits crossing, etc. Over secular timescales, some embryos may collide, while some may be ejected from the system. This chaotic growth characterizes the final stage of planet formation, and sets the mass, composition, and orbital architecture of the planetary system.

The planetesimal accretion scenario suffers from a major difficulty when applied to the formation of giant planet cores towards larger separation. In the case of Jupiter and Saturn at $a \sim 5-10 \mathrm{AU}$, reduction of $\Sigma_{\mathrm{p}}$ and increase of the dynamical time at larger radii make the growth timescale substantially longer than in the inner disk, as can be seen from Eq. (6). Correspondingly, the timescale to build up a sufficiently massive core $\left(\sim 10-15 M_{\oplus}\right.$, Pollack et al. 1996) to trigger runaway accretion of a gaseous envelope would well exceed the typical lifetime of a protoplanetary disk ( $\sim 3$ Myrs, Haisch et al. 2001). This issue can be alleviated when considering accretion of smaller planetesimals (Rafikov 2004), enhancement of $\Sigma_{\mathrm{p}}$ by a factor of a few beyond the snow line (e.g., Kennedy and Kenyon 2008), and probably resolved when type-I migration of the embryos is considered (Alibert et al. 2005). In the latter scenario, orbital migration via planet-disk interaction allows the embryos to sweep up planetesimals over the course of their migration, and avoids severe depletion of the feeding zone. Incorporating inward planet migration into planet population synthesis models (e.g., Ida and Lin 2008; Mordasini et al. 2009a), it is found that, while forming giant planets at the location of Jupiter and Saturn is plausible, the overall outcome depends sensitively on the strength of planetary migration, which remains to be better understood (see Sect. 3).

Recently, a number of giant planets have been discovered via direct imaging surveys to reside at large orbital separations. Notable examples include $\beta$-Pic $b$ (Lagrange et al. 2010) and the HR 8799 system (Marois et al. 2010), where orbital separation reaches up to $\sim 70$ AU. The conventional core accretion theory via planetesimal accretion simply fails in such cases due to the rapid decline of planetesimal density and rapid increase of dynamical timescale towards large orbital distances. One possible alternative to form giant planets at large orbital separation is by the gravitational instability (GI, Boss 1997), which likely operates in the outer disk during the early stages of disk evolution. We will discuss GI further in Sect. 4.2.

\subsection{Pebble Accretion}

Recently, a new model of planetesimal/core growth has been proposed, where it was found that accretion of mm- to cm-sized grains, or pebbles, can be a much more efficient mode of core growth (Lambrechts and Johansen 2012; Ormel and Klahr 2010; Johansen and Lacerda 2010; Perets and Murray-Clay 2011; Morbidelli and Nesvorny 2012). A large fraction of protoplanetary disks have been observed to contain a significant fraction of dust mass in mm-sized grains (Natta et al. 2007; Ricci et al. 2010), some also reveal the presence of $\mathrm{cm}$ sized pebbles (Testi et al. 2003; Wilner et al. 2005; Rodmann et al. 2006). These solids couple strongly with disk gas via aerodynamic drag, the relative velocity can be strongly 
damped during gravitational encounters, leading to substantially enhanced cross section. Because of its relative novelty, we devote more effort discussing this new picture of pebble accretion.

\subsubsection{Pebble Aerodynamics}

Due to partial pressure support, the gas in protoplanetary disks rotates at a velocity slightly slower than the Keplerian velocity. The velocity difference, denoted by $\Delta v_{\mathrm{K}}$, is typically a very small fraction of the Keplerian velocity $\left(\sim 10^{-3}-10^{-2}\right.$ depending on location), and is commonly normalized by the local sound speed $c_{\mathrm{s}}$. In the standard MMSN disk, it is given by $\Delta v_{\mathrm{K}} \approx 0.1(R / 10 \mathrm{AU})^{1 / 4} c_{\mathrm{s}}$ at disk midplane.

The pressureless dust grains, on the other hand, tend to follow Keplerian orbits. They thus experience a headwind from the gas and drift radially inward. The aerodynamic friction between gas and pebbles is characterized by the stopping time $t_{s}$, which is commonly normalized to the orbital time to give $\tau_{s} \equiv \Omega_{\mathrm{K}} t_{s}$. This dimensionless stopping time is also called the Stokes number. Strong and weak aerodynamic coupling correspond to $\tau_{s} \ll 1$ and $\tau_{s} \gg 1$, respectively. For an MMSN disk, a spherical grain with size $s$ has a stopping time in the disk midplane (in the Epstein regime) equal to

$$
\tau_{s}=0.14\left(\frac{s}{1 \mathrm{~cm}}\right)\left(\frac{R}{10 \mathrm{AU}}\right)^{3 / 2} .
$$

Thus, pebble-sized grains in the outer disk are close to be marginally coupled with the gas. Assuming pebbles are passive in a laminar disk, particles experience a radial drift, with sub-Keplerian rotation, whose velocity components are given by

$$
v_{r}=-\frac{2 \tau_{s}}{1+\tau_{s}^{2}} \Delta v_{\mathrm{K}}, \quad v_{\phi}-v_{\mathrm{K}}=-\frac{1}{1+\tau_{s}^{2}} \Delta v_{\mathrm{K}},
$$

where $v_{\mathrm{K}}$ is the Keplerian velocity. Radial drift is most efficient for marginally coupled particles with $\tau_{s} \sim 1$. In any case, the difference between the particles velocity and the local Keplerian velocity is still on the order of $\Delta v_{\mathrm{K}}$.

In the vertical direction, particles experience vertical gravity $g_{z}=-\Omega_{\mathrm{K}}^{2} z$, leading to settling towards the disk midplane. This is balanced by turbulent diffusion. Denoting by $D_{\mathrm{p}, \mathrm{z}}$ the particles diffusion coefficient in the vertical direction, particles tend to adopt a Gaussian density profile of the form $\rho_{\mathrm{p}}=\rho_{\mathrm{p} 0} e^{-z^{2} / 2 H_{\mathrm{p}}^{2}}$ that is characterized by a particle scale height

$$
H_{\mathrm{p}} \approx \sqrt{\frac{D_{\mathrm{p}, \mathrm{z}}}{\Omega_{\mathrm{K}} \tau_{s}}}
$$

The turbulent diffusion coefficient of the gas, $D_{\mathrm{g}}$, can be generally written as $D_{\mathrm{g}} \sim \delta v_{\mathrm{g}}^{2} \times$ $\tau_{\text {corr }}$, where $\delta v_{\mathrm{g}}$ is the turbulent velocity of the gas, and $\tau_{\text {corr }}$ is the correlation time of the gas turbulence, which is expected to be on the order of $\Omega_{\mathrm{K}}^{-1}$. The particles' diffusion coefficient $D_{\mathrm{p}}$ is generally comparable to $D_{\mathrm{g}}$ for marginally to strongly coupled particles $\tau_{s} \lesssim 1$ (Youdin and Lithwick 2007). The level of turbulence in protoplanetary disks is uncertain, but is expected to be weak (e.g., Bai 2015). For $\delta v_{\mathrm{g}} \sim 10^{-2} c_{\mathrm{s}}$, we have $D_{\mathrm{g}} \approx D_{\mathrm{p}} \sim 10^{-4} c_{\mathrm{s}} H_{\mathrm{g}}$, with $H_{\mathrm{g}}$ the gas pressure scale height $\left(H_{\mathrm{g}}=c_{\mathrm{s}} / \Omega_{\mathrm{K}}\right)$. The particles scale height is therefore $H_{\mathrm{p}} \sim 0.01 H_{\mathrm{g}}$ for $\tau_{s}=1$, and $H_{\mathrm{p}} \sim 0.1 H_{\mathrm{g}}$ for $\tau_{s}=0.01$. 


\subsubsection{Two Regimes of Pebble Accretion}

Accurate determination of pebble accretion rates requires detailed analysis of particle orbits as they approach the planetesimals/embryos (e.g., Ormel and Klahr 2010), and the orbits can become fairly complex especially for loosely coupled large solids $\left(\tau_{s}\right.$ well above 1$)$. For more tightly coupled particles $\left(\tau_{s} \lesssim 1\right)$, which are relevant in the pebble accretion scenario, simple order-of-magnitude analysis proves to be sufficient, as we describe here.

We consider particle trajectories as they approach the planetesimal/core in the background gas. The gas is assumed to be unperturbed by the core. ${ }^{1}$ Therefore, if particles are very strongly coupled to the gas, they are largely entrained by the gas without being accreted. More generally, particles can decouple from the gas on timescales of their stopping time $\sim t_{s}$. They can be accreted if strong gravitational deflection can be achieved within $t_{s}$, which depends on the impact parameter $r$. This is the basic physics of pebble accretion. Key in calculating the pebble accretion rate is to estimate the maximum impact parameter $\left(r_{a}\right)$ or the accretion radius, within which pebbles can be accreted.

Before calculating the rate of pebble accretion, it is useful to distinguish two regimes of pebble accretion, determined by the mass $M_{c}$ of the planetesimal/core. To begin with, we can conveniently define a Bondi radius as ${ }^{2}$

$$
R_{\mathrm{B}}=\frac{G M_{c}}{\Delta v_{\mathrm{K}}^{2}} .
$$

This definition of $R_{\mathrm{B}}$ marks the length scale at which pebbles can be significantly deflected by the planetesimal/core by two-body interactions. Additionally, this deflection can not be effective beyond the Hill radius $R_{\mathrm{H}}$ (Eq. (4)), where three-body effects become important and the core loses gravitational dominance.

By equating $R_{\mathrm{B}}$ and $R_{\mathrm{H}}$, the transition mass can be defined as

$$
M_{t}=\frac{\Delta v_{\mathrm{K}}^{3}}{G \Omega_{\mathrm{K}}} \approx 0.16 M_{\oplus}\left(\frac{R}{30 \mathrm{AU}}\right)^{3 / 4}\left(\frac{\Delta v_{\mathrm{K}}}{0.1 c_{\mathrm{s}}}\right)^{3},
$$

where in the second equality we use the MMSN scaling for the sound speed. Note that $R_{\mathrm{B}} \propto M_{c}$ while $R_{\mathrm{H}} \propto M_{c}^{1 / 3}$, thus $\left(R_{\mathrm{B}} / R_{\mathrm{H}}\right) \approx\left(M_{c} / M_{t}\right)^{2 / 3}$. Therefore, the distance from the planetesimal/core within which its gravitational influence is effective is given by $R_{\mathrm{B}}$ for low-mass cores, and by $R_{\mathrm{H}}$ for high-mass cores. Correspondingly, the transition mass defined above separates the two regimes of pebble accretion, which are termed drift regime for $M_{c}<M_{t}$, and Hill regime for $M_{c}>M_{t}$. Estimates of the accretion radius $r_{a}$ differ between both regimes, as we address below.

\subsubsection{Drift Regime}

In the drift regime where $M_{c}<M_{t}$ (or, equivalently, the Bondi radius $R_{\mathrm{B}}<R_{\mathrm{H}}$ ), the accretion radius $r_{a} \leq R_{\mathrm{B}}$. Within this radius, particles approach the core with relative velocity $\Delta v \sim \Delta v_{\mathrm{K}}$. The timescale for a particle to pass by the core is

$$
t_{B} \approx \frac{R_{\mathrm{B}}}{\Delta v_{\mathrm{K}}}=\frac{1}{\Omega_{\mathrm{K}}} \frac{M_{c}}{M_{t}} .
$$

\footnotetext{
${ }^{1}$ As long as $M_{C}$ is much smaller than the "thermal mass" $M_{\mathrm{th}}=c_{\mathrm{S}}^{3} / G \Omega_{\mathrm{K}}$, which is about the mass scale of Jupiter and is well beyond the mass scale for core growth.

${ }^{2}$ Note this definition given in Lambrechts and Johansen (2012) is different from the conventional definition of the Bondi radius, where $\Delta v_{\mathrm{K}}$ is replaced by the sound speed.
} 
Particles with stopping time $t_{s} \approx t_{B}$ decouple from the gas during the encounter, and are expected to spiral into the core once their impact parameter is within $R_{\mathrm{B}}$. Therefore, the accretion radius $r_{a} \approx R_{\mathrm{B}}$ for $t_{s} \approx t_{B}$.

For more strongly coupled particles with $t_{s}<t_{B}$, they can only be captured when their impact parameter is smaller than $R_{\mathrm{B}}$, so that they experience stronger gravity with shorter gravitational deflection time (and hence they can decouple from the gas and spiral in). Otherwise, they will be entrained by the gas flow without being accreted. The gravitational deflection time for given impact parameter $r$ is

$$
t_{g}(r) \approx \frac{\Delta v_{\mathrm{K}}}{G M_{c} / r^{2}}=\left(\frac{r}{R_{\mathrm{B}}}\right)^{2} t_{B} .
$$

By equating $t_{g}(r)$ with $t_{s}$, the accretion radius $r_{a}$ can be obtained as

$$
r_{a} \approx\left(\frac{t_{s}}{t_{B}}\right)^{1 / 2} R_{\mathrm{B}} \approx \tau_{s}^{1 / 2}\left(\frac{M_{c}}{M_{t}}\right)^{1 / 6} R_{\mathrm{H}} .
$$

This radius can equivalently be understood as the radius at which the gravitational acceleration is equal to the acceleration due to gas drag (also termed as the wind-shearing radius, Perets and Murray-Clay 2011).

For more weakly coupled particles with $t_{s}>t_{B}$, they behave more similarly to the gasfree scenario, where particles follow hyperbolic orbits as they encounter the core. Instead of the Bondi radius, it is the physical size of the core (much smaller) that determines whether accretion takes place. This results in a rapid decline of $r_{a}$ as $t_{s}$ increases beyond $t_{B}$, and it becomes negligible compared with the $t_{s} \sim t_{B}$ case.

We note that in the drift regime, $\Omega_{\mathrm{K}} t_{B} \approx M_{c} / M_{t}$. Therefore, efficient accretion of dust grains in the drift regime always takes place for particles with $\tau_{s}<1$. The smaller the core mass, the smaller the optimal particle size.

\subsubsection{Hill Regime}

In the Hill regime where $M_{c}>M_{t}$ (or, equivalently, the Bondi radius $R_{\mathrm{B}}>R_{\mathrm{H}}$ ), we expect the accretion radius $r_{a} \leq R_{\mathrm{H}}$. Within this radius, the relative velocity with which particles approach the core can be dominated by the Keplerian shear, $\Delta v_{\mathrm{sh}}(r) \equiv(3 / 2) \Omega_{\mathrm{K}} r$. To see this, note that

$$
\frac{\Delta v_{\mathrm{sh}}(r)}{\Delta v_{\mathrm{K}}}=\frac{3}{2}\left(\frac{M_{c}}{M_{t}}\right)\left(\frac{r}{R_{\mathrm{B}}}\right) .
$$

Therefore, the shear velocity dominates for impact parameters $r \gtrsim R_{\mathrm{B}}$. For a given impact parameter $r$, the relative velocity is $\Delta v(r) \approx \max \left[\Delta v_{\mathrm{K}}, \Delta v_{\mathrm{sh}}(r)\right]$.

Following the same spirit as before, we write the gravitational deflection time for particles as

$$
t_{g}(r)=\frac{\Delta v}{G M_{c} / r^{2}} \approx \frac{\Omega_{\mathrm{K}} r}{G M_{c} / r^{2}} \approx \frac{1}{\Omega_{\mathrm{K}}}\left(\frac{r}{R_{\mathrm{H}}}\right)^{3},
$$

where we have taken $\Delta v \approx \Omega_{\mathrm{K}} r$. Efficient pebble accretion requires $t_{g} \lesssim t_{s}$, which leads to an accretion radius of

$$
r_{a} \approx\left(\Omega_{\mathrm{K}} t_{s}\right)^{1 / 3} R_{\mathrm{H}}=\tau_{s}^{1 / 3} R_{\mathrm{H}}
$$


This result again holds ${ }^{3}$ for particles with $\tau_{s} \lesssim 1$. Particles with lager stopping times would undergo 3-body scattering whose orbits can become fairly chaotic. While gas drag still enhances particle accretion, accretion is less efficient with increasing $\tau_{s}$, and more over, we do not expect a large population of particles with stopping times far exceeding 1 to be present in protoplanetary disks.

The above scaling needs to be revised when $r_{a}<R_{\mathrm{B}}$, at which point we should have taken $\Delta v=\Delta v_{\mathrm{K}}$. The revised $r_{a}$ then becomes the expression in Eq. (14). Therefore, the overall result is

$$
r_{a} \approx \min \left[\tau_{s}^{1 / 2}\left(\frac{M_{c}}{M_{t}}\right)^{1 / 6}, \tau_{s}^{1 / 3}\right] R_{\mathrm{H}} .
$$

The fact that the accretion radius approaches the Hill radius in the Hill regime makes pebble accretion an extremely efficient mechanism for planetesimal/core growth. Additionally, the weak dependence of the accretion radius on $\tau_{s}$ in Eq. (18) indicates efficient accretion takes place for a wide range of particle sizes (say $\tau_{s}=0.01-1$ ).

\subsubsection{Timescale for Core Growth}

The rate of pebble accretion is determined by the accretion radius $r_{a}$, and the radial flux of pebbles approaching the core. To calculate it, we need to distinguish the 2D and 3D cases, depending on whether the particles' scale height $H_{\mathrm{p}}$ is larger or smaller than $r_{a}$. In the following, we denote by $\Sigma_{\mathrm{p}}$ the surface density of the pebbles.

When $H_{\mathrm{p}}<r_{a}$, we are in the $2 \mathrm{D}$ regime where particles settle strongly to the midplane so that the entire particle column approaching the core can be accreted, with accretion rate

$$
\dot{M}_{2 D} \approx 2 \Sigma_{\mathrm{p}} r_{a} \Delta v
$$

When $H_{\mathrm{p}} \gtrsim r_{a}$, we are in the 3D regime where particles are suspended, and only those close to the midplane with $|z| \lesssim r_{a}$ can be accreted. The accretion rate is given by

$$
\dot{M}_{3 D} \approx \frac{\Sigma_{\mathrm{p}}}{H_{\mathrm{p}}} \pi r_{a}^{2} \Delta v .
$$

In both cases, we have $\Delta v \approx \max \left[\Delta v_{\mathrm{K}}, \Delta v_{\mathrm{sh}}\left(r_{a}\right)\right]$.

Now we can discuss the planetesimal/core growth rate due to pebble accretion. While multiple combinations of 2D/3D and drift/Hill regimes are possible, here we simply discuss two most representative cases.

In the early stage of planet formation when the planetesimal/core is small, pebble accretion is in the drift regime with $R_{\mathrm{B}}<R_{\mathrm{H}}$, and likely proceeds in $3 \mathrm{D}$. In that case, we get

$$
\dot{M}_{c} \approx \pi \frac{\Sigma_{\mathrm{p}}}{H_{\mathrm{p}}}\left(\frac{t_{s}}{t_{B}} R_{\mathrm{B}}^{2}\right) \Delta v_{\mathrm{K}} .
$$

In the optimistic case where the contribution from $t_{s} / t_{B} \sim 1$ particles is considered ( $t_{s}$ increases with $M_{c}$ ), growth can achieve super-runaway with $\dot{M}_{c} \sim M_{c}^{2}$, although the base rate (i.e., proportional coefficient) can be very low.

\footnotetext{
${ }^{3}$ By integrating individual particle trajectories, $r_{a}$ is found to be about $R_{\mathrm{H}}$ for particles with $\tau_{s} \sim 0.1$ (Lambrechts and Johansen 2012), thus $r_{a} \approx\left(\tau_{s} / 0.1\right)^{1 / 3} R_{\mathrm{H}}$ is probably more accurate for $\tau_{s} \lesssim 0.1$, which still agrees with the order-of-magnitude derivation within a factor of order unity.
} 
Fig. 1 Core growth as a function of time for various orbital distances for the pebble accretion scenario in the Hill and drift regimes, as well as the conventional planetesimal accretion (denoted by "PA"), taken from Lambrechts and Johansen (2012). This calculation assumes parameter $\Delta \equiv \Delta v_{\mathrm{K}} / c_{\mathrm{S}}=0.05$, and dust-to-gas mass ratio $Z=0.01$ in a minimum-mass solar nebular disk

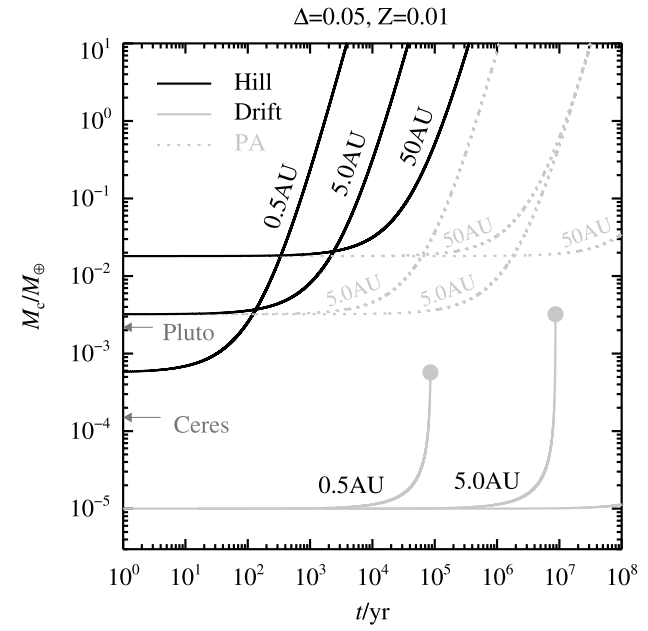

Towards later stages of planet formation, when the core has grown substantially across the transition mass, accretion proceeds in the Hill regime and can operate in $2 \mathrm{D}$ if turbulence is not too strong. The corresponding accretion rate is

$$
\dot{M}_{c} \approx 2 \Sigma_{\mathrm{p}} \tau_{s}^{2 / 3} R_{\mathrm{H}}^{2} \Omega_{\mathrm{K}}
$$

In terms of scaling, we obtain $\dot{M}_{c} \propto M_{c}^{2 / 3}$, which is the same rate as with oligarchic growth. However, the proportional coefficient is much larger than in the planetesimal accretion scenario, because of the greatly enhanced cross section of the order $R_{\mathrm{H}}^{2}$.

To evaluate the core growth timescale via pebble accretion (in the Hill regime), we first rewrite Eq. (22) into the more intuitive form

$$
\frac{1}{M_{c}} \frac{d M_{c}}{d t} \approx \frac{\Sigma_{\mathrm{p}} r^{2}}{M_{*}}\left(\frac{M_{*}}{M_{c}}\right)^{1 / 3} \Omega_{\mathrm{K}},
$$

where we consider the most optimistic case that all particles entering the Hill sphere get accreted. This roughly corresponds to the case where most dust particles have stopping time $\tau_{s} \sim 0.1-1$ (and 2D accretion), which is probably roughly satisfied in the outer regions of protoplanetary disks. We note that the growth rate is independent of $\Delta v_{\mathrm{K}}$ (but the transition mass does). Assuming a Solar mass star, the growth time is

$$
t_{\text {grow }} \approx 2.2 \times 10^{3}\left(\frac{2 \pi}{\Omega_{\mathrm{K}}}\right)\left(\frac{0.005 M_{\odot}}{\Sigma_{\mathrm{p}} r^{2}}\right)\left(\frac{M_{c}}{M_{\oplus}}\right)^{1 / 3} .
$$

Assuming a typical disk mass of $\sim 0.01 M_{\odot}$, and that the planet is formed half-way, then for an earth-mass core the growth time is about 2000 orbits, which is only $\sim 3.6 \times 10^{5}$ years at $30 \mathrm{AU}$.

Figure 1 shows a simple model for the time evolution of the core's mass resulting from the conventional planetesimal accretion as well as from pebble accretion, taken from Lambrechts and Johansen (2012). The calculations correspond to the optimistic 2D accretion case with maximum accretion rate. The general conclusions are that pebble accretion in the Hill regime is extremely efficient, especially at large orbital distances. On the other hand, in the drift regime, pebble accretion is slow and core growth generally proceeds more efficiently via planetesimal accretion. 


\subsubsection{Global Models and Uncertainties}

The general formulation of pebble accretion has recently been incorporated in global models of planet formation (Chambers 2014; Kretke and Levison 2014; Lambrechts and Johansen 2014; Bitsch et al. 2015a). In general, these models confirm that pebble accretion is indeed very efficient and can well account for the formation of the cores of not only the gas and ice giant planets in the Solar system, but also the extrasolar giant planets at large separations. The growth might be fast enough to avoid large-scale type I planetary migration (see Sect. 3.1.3). Additionally, a large fraction $(\sim 50 \%)$ of pebbles undergoing radial drift can be intercepted by the embryos/planetesimals, leading to very efficient conversion of solids into planets (Lambrechts and Johansen 2014). On the other hand, formation of gas giants is less favored in systems with relatively small disk masses and/or metallicities, consistent with the metallicity trend in the occurrence rate of giant exoplanets (Fischer and Valenti 2005; Bitsch et al. 2015a; Johnson et al. 2010).

Applying to the Solar system, criticisms arise concerning the fact that it may be too efficient: the system tends to form a large number of planetary embryos inside-out, which would lead to violent dynamical interactions and can hardly be made consistent with the planetary architecture of the inner Solar system (Kretke and Levison 2014). On the other hand, Morbidelli et al. (2015) pointed out that pebble accretion is likely very inefficient within the snow line due to reduced grain size, which readily explains the dichotomy of low-mass terrestrial planets in the inner Solar system and gas giants in the outer parts.

At the present stage, studies from different groups have adopted different methodologies in treating various physical processes during planetesimal/core growth, and inevitably involve simplified assumptions in one way or another. As also noticed in these works, major uncertainties exist in these modeling efforts, which include: (1) The size distribution and evolution of pebbles, which were either treated as the end product of grain growth process, or as fragments from planetesimal collision. (2) The initial mass function of planetesimals, the starting point of planetesimal growth. (3) Global disk structure and evolution, and level of turbulence, particularly relevant for the dynamics and transport of pebbles. None of these factors are well understood, and they are interrelated with each other.

The key to unravel these uncertainties probably lies in better understanding the internal structure and evolution of protoplanetary disks from first principle. Recent local 3D MHD simulations including realistic non-ideal MHD physics have suggested a paradigm shift from the conventional viscous evolution picture towards a magnetized disk wind driven evolution of the inner disk (Bai and Stone 2013; Gressel et al. 2015), and a weakly turbulent outer disk with substructures due to magnetic flux concentration (Bai and Stone 2014; Bai 2015). Extension of these local studies to global simulations is essential and will likely help establish a most realistic picture of protoplanetary disks. Such a picture will provide essential input for both grain growth calculations and necessary conditions for planetesimal formation (see chapter by Birnstiel et al.), and altogether, for modeling the entire processes of planet formation.

\section{Orbital Evolution of Planets in Their Protoplanetary Disk}

As planets form in their protoplanetary disk, the gravitational interaction between the disk and the planets change the semi-major axis, eccentricity and inclination of the planets. Planet-disk interactions usually damp eccentricities and inclinations efficiently, but as we will see throughout this section, they can either decrease or increase the planets semi-major 
axes, an effect usually termed planetary migration or disk migration. Planet-planet interactions tend to pump eccentricities and inclinations, and can significantly change semi-major axes during the disk's lifetime, for example through scattering events due to the disk migration of two or more planets. After the protoplanetary disk is cleared by photo-evaporation, by typically 1 to 10 Myrs, the orbital evolution of planets can continue through interaction with the central star (via tides or stellar evolution for close-in planets), with distant stars (e.g., via Kozai cycles), with a disk of remnant planetesimals (debris disk), or via planet-planet interactions.

As this chapter is about the early evolution of planetary systems, we will focus on the orbital evolution of planets driven by planet-disk interactions. The long-term evolution of planetary systems, after dispersal of the protoplanetary disk, will be presented in the chapter by Zhou et al. The aim of this section is to give a simple, practical overview of the physical processes that drive planet-disk interactions. The case of low-mass planets (typically Earthmass planets) is described in Sect. 3.1, that of massive planets (typically Jovian planets) follows in Sect. 3.2. We then discuss in Sect. 3.3 what models of planet-disk interactions can and cannot explain about hot Jupiters (Sect. 3.3.1), and about the many super-Earths in multi-planet systems discovered by Kepler (Sect. 3.3.2). More in-depth reviews of planetdisk interactions can be found in Kley and Nelson (2012), Baruteau and Masset (2013) and Baruteau et al. (2014).

\subsection{Disk Migration of Low-Mass Planets}

Since planetary migration is driven by the gravitational interaction between a planet and its parent protoplanetary disk, the basics of planetary migration can be drawn through inspection of the gas density perturbation caused by a protoplanet. Figure 2 shows the relative perturbation of the gas surface density of a protoplanetary disk where a 5 Earth-mass planet forms. The figure is taken from a 2D hydrodynamical simulation of planet-disk interactions for a non-magnetized radiative disk model. As can be seen in the figure, the planet induces two kinds of density perturbations in the disk: (i) spiral density waves, called the planet's wakes, which propagate throughout the disk, and (ii) density perturbations very close to the planet's orbital radius, called co-orbital density perturbations, which are confined in the planet's horseshoe region (see right panel), where gas describes horseshoe trajectories relative to the planet.

The basic idea underlying planet-disk interactions is the law of action-reaction: the disk reacts to the planet's gravity by exerting a gravitational force on the planet which changes the planet's semi-major axis, eccentricity and inclination. Considering the simplest case of a planet on a circular and coplanar orbit (with zero eccentricity and inclination), the planet's angular momentum is $J_{\mathrm{p}}=M_{\mathrm{p}} \sqrt{G M_{\star} r_{\mathrm{p}}}$, where $M_{\mathrm{p}}$ is the mass of the planet, $M_{\star}$ that of the star, $G$ is the gravitational constant, and $r_{\mathrm{p}}$ is the distance between the star and the planet. Denoting by $\Gamma$ the torque exerted by the disk on the planet, and further assuming the planet mass is stationary, $\Gamma=d J_{\mathrm{p}} / d t$ implies $d r_{\mathrm{p}} / d t=\Gamma \times 2 r_{\mathrm{p}} / J_{\mathrm{p}}$. This relation simply means that, in order to know the direction and speed of planetary migration, the sign and magnitude of the disk torque need to be determined. This is why most numerical studies of planet-disk interactions take planets on fixed orbits and calculate the disk torque on the planet. However, as we will see later, the disk torque can actually be a function of the migration rate and migration history, with the consequence that, under some circumstances, the migration can runaway. We briefly outline below the different components of the disk torque. 


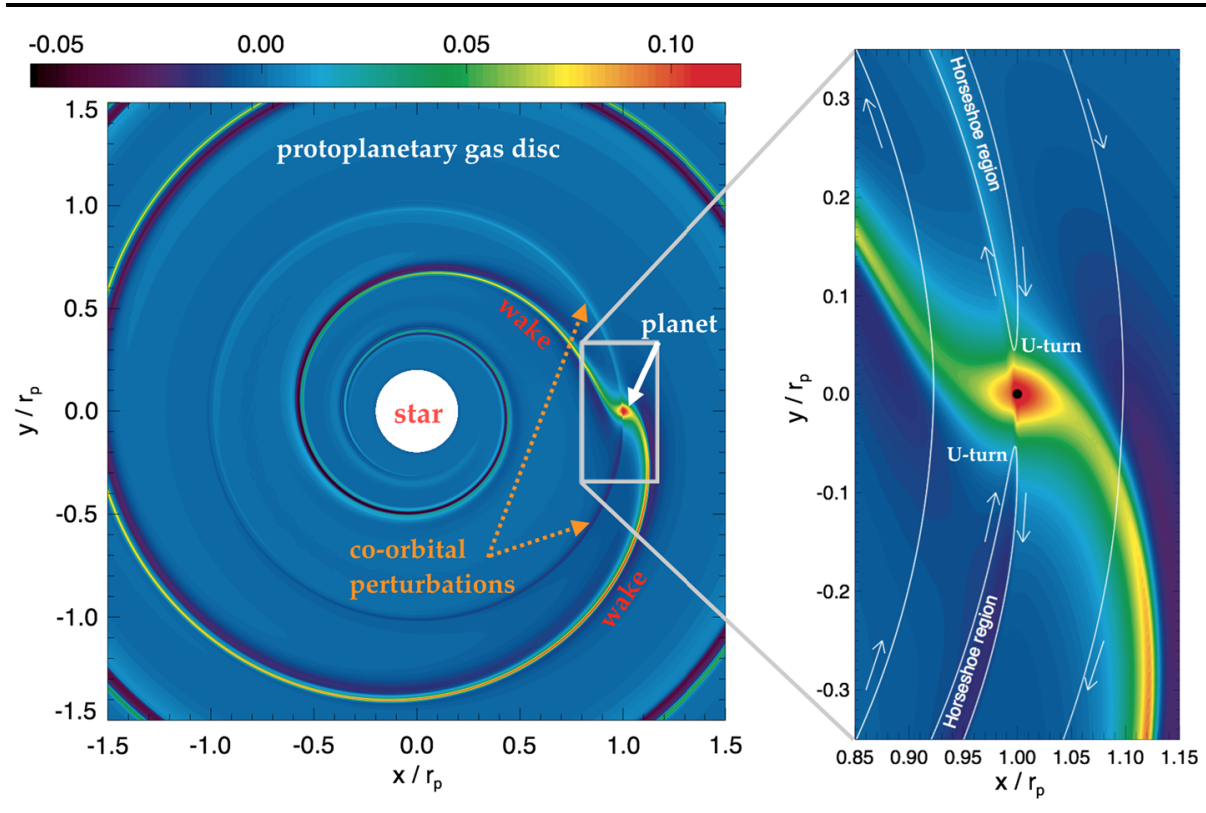

Fig. 2 Relative perturbation of the gas surface density of a protoplanetary disk perturbed by a 5 Earth-mass planet. The planet's location is spotted by the white arrow in the left panel. The planet generates a one-armed spiral density wave - the wake - that propagates throughout the disk, as well as co-orbital density perturbations within the planet's horseshoe region. Gas trajectories with respect to the planet are depicted by white curves and arrows in the right panel. Figure adapted from Baruteau et al. (2014)

\subsubsection{Wake Torque}

First, there is the torque due to the inner wake, which is the wake that propagates between the planet and the star. Most of the inner wake's torque comes from the excess of gas just in front of the planet in the azimuthal direction, and which is distant from the planet by a few pressure scale heights at most. This gas excess exerts a gravitational force on the planet whose azimuthal component $F_{\varphi}$ is positive, which corresponds to a torque $\sim r_{\mathrm{p}} F_{\varphi}$ on the planet that is therefore positive. Similarly, the outer wake's torque is mostly due to the excess of gas immediately behind the planet in the azimuthal direction. That local gas excess now has $F_{\varphi}<0$ and thus exerts a negative torque on the planet. The total wake torque then has two opposite contributions: the (positive) torque due to the inner wake that tends to move the planet outwards, and the (negative) torque due to the outer wake that tends to move the planet inwards. Inspection of the inset panel in the right-hand side of Fig. 2 shows that the gas density perturbation in the outer wake is slightly larger and closer to the planet, which indicates that the outer wake's torque is stronger. The total wake torque is therefore negative in that case and favors inward migration. While this is indeed the general expectation, the reader should be aware that this result depends on the radial gradients of the gas temperature and density near the planet's location, as they affect the location of the wakes relative to the planet as well as their density enhancement. These dependencies can be found by solving the linear perturbation equations numerically, either in 2D (Korycansky and Pollack 1993; Ward 1997; Paardekooper et al. 2010; Masset 2011) or in 3D (Tanaka et al. 2002). The reader is referred to Sect. 2.1.1 of Baruteau et al. (2014) for more details on the wake torque and its expression as a function of disk gradients. 


\subsubsection{Corotation Torque}

The disk gas in the planet's horseshoe region follows horseshoe trajectories as seen from the planet. At its closest approach to the planet, the gas undergoes a gravitational kick from the planet. The gas just behind the planet in the azimuthal direction and inside the planet's orbital radius moves radially outward by taking angular momentum from the planet. This gas takes outward U-turns relative to the planet and exerts a negative torque on the planet. Meanwhile, gas just in front on the planet in the azimuthal direction and outside the planet's orbital radius moves radially inward by giving angular momentum to the planet. That gas embarks on inward U-turns and exerts a positive torque on the planet. Much like the wake torque, the torque exerted by the horseshoe region, which is called the corotation torque or horseshoe drag, has two opposite contributions. The sign and magnitude of the corotation torque then depend on the angular momentum difference between gas doing inward and outward U-turns.

Complexity arises as the angular momentum of the gas in the planet's horseshoe region evolves in time due to the advection-diffusion of two hydrodynamical quantities: (i) the gas specific vorticity, sometimes called vortensity, which in $2 \mathrm{D}$ is the vertical component of the velocity curl (vorticity) divided by the surface density, and (ii) the gas specific entropy, which in $2 \mathrm{D}$ is basically the quantity $P \Sigma^{-\gamma}$ with $P$ the thermal pressure, $\Sigma$ the surface density and $\gamma$ the adiabatic index. Advection of vortensity and specific entropy along horseshoe streamlines implies that the sign and magnitude of the corotation torque depend on the density and temperature gradients across the horseshoe region. Diffusion of both quantities implies that the corotation torque also depends on the nature and efficiency of turbulent diffusion mechanisms taking place near or inside the horseshoe region, which for low-mass planets is a small fraction of the disk's pressure scale height. Modeling of turbulence by a viscosity and a thermal diffusivity shows that the corotation torque is very sensitive to both diffusion parameters. Hence the importance of better understanding how turbulent transport of angular momentum operates in regions of planet formation (e.g., Lesur et al. 2014; Bai 2015; Gressel et al. 2015). 2D and 3D models of viscous disks show that the corotation torque is usually positive and therefore favors outward migration (e.g., Paardekooper and Mellema 2006; Baruteau and Masset 2008; Paardekooper and Papaloizou 2008; Kley and Crida 2008; Kley et al. 2009; Masset and Casoli 2010; Paardekooper et al. 2010, 2011; Lega et al. 2014). The reader is referred to Sect. 2.1.2 of (Baruteau et al. 2014) for more details on the corotation torque. Its expression in $2 \mathrm{D}$ as a function of disk gradients, viscosity and thermal diffusivity can be found in Masset and Casoli (2010) or Paardekooper et al. (2011). A derivation of the corotation torque in 3D globally isothermal disk models has been recently presented by Masset and Benítez-Llambay (2016). Dynamical corotation torques, which arise as the planet drifts relative to the disk, will be described in Sect. 3.1.4.

\subsubsection{Type I Migration}

The migration of low-mass planets (typically up to Neptune's mass), known as type I migration, is driven by the sum of the wake torque (Sect. 3.1.1) and the corotation torque (Sect. 3.1.2). The former is usually negative, the latter usually positive. Once more, we have two competing effects, and the balance between them is very sensitive to the disk model. This is illustrated in Fig. 3, which displays the type I migration torque with varying planet mass and planet's orbital radius. In this disk model taken from Bitsch et al. (2013b), opacity transitions near the evaporation line of silicates (at $r \approx 0.7 \mathrm{AU}$ ) and the water ice line (at $r \approx 5 \mathrm{AU}$ ) boost the corotation torque beyond these transitions, where a broad range 
Fig. 3 Type I migration torque on planets of different masses (y-axis) and orbital radii ( $x$-axis). Horizontal arrows show the direction of migration. Figure adapted from Bitsch et al. (2013b), where the disk model sets the water ice line at $r \approx 5$ $\mathrm{AU}$, and the evaporation line of silicates at $r \approx 0.7 \mathrm{AU}$

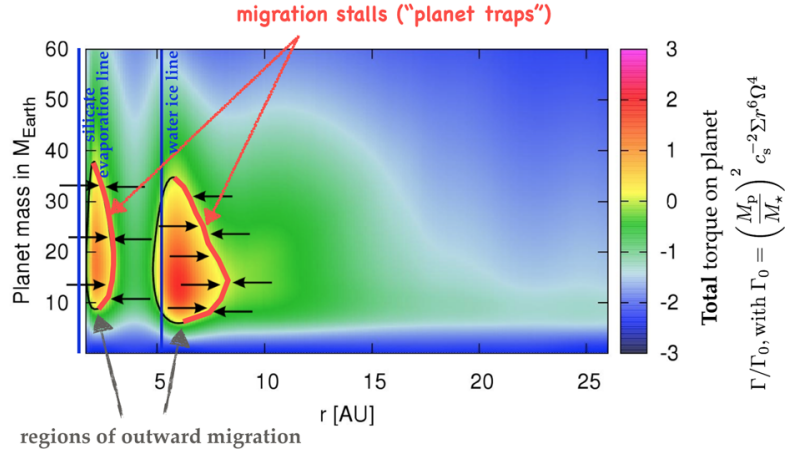

migration stalls ("planet traps")

of planet masses undergoes outward migration. Note that type I migrating cores converge towards the outer edge of these regions of outward migration; these locations are known as "planet traps".

The torque value in Fig. 3 can be used to estimate the type I migration timescale. The factor $\Gamma_{0}$ by which the torque is normalized in the color bar sets indeed a typical timescale for type I migration, $\tau_{0}$, given by $\tau_{0}=\Omega_{\mathrm{K}} M_{\mathrm{p}} r_{\mathrm{p}}^{2} / 2 \Gamma_{0}$, and which can be recast as

$$
\tau_{0} \approx 1.3 \mathrm{Myr} \times\left(\frac{h}{0.05}\right)^{2}\left(\frac{\Sigma}{200 \mathrm{~g} \mathrm{~cm}^{-2}}\right)^{-1}\left(\frac{M_{\star}}{M_{\odot}}\right)^{3 / 2}\left(\frac{r_{\mathrm{p}}}{5 \mathrm{AU}}\right)^{-1 / 2}\left(\frac{M_{\mathrm{p}}}{M_{\oplus}}\right)^{-1},
$$

with $h=c_{\mathrm{S}} / v_{\mathrm{K}}$ the disk's aspect ratio, and where all gas quantities are to be calculated at the planet's orbital radius. Above estimate shows that an Earth-mass planet located at 5 AU from a Solar-mass star, with local gas surface density of $200 \mathrm{~g} \mathrm{~cm}^{-2}$ and disk's aspect ratio of $5 \%$, and which experiences a total disk torque $\Gamma \approx-2 \Gamma_{0}$ (see Fig. 3), would migrate inwards on a characteristic timescale of $\sim 0.7 \mathrm{Myr}$. This should be compared with the typical growth timescale of the planet. In the Hill regime of pebble accretion, the growth timescale of such a planet could be as short as $\sim 2 \times 10^{4} \mathrm{yr}$ (see the black bold curves in Fig. 1), which suggests that Earth-mass planets could grow fast enough to avoid significant inward migration and reach planetary traps (the red bold curves in Fig. 3). Planetesimal accretion, however, would result in a growth timescale at least comparable to the aforementioned migration timescale (see the dashed curves in Fig. 1), in which case significant inward migration would occur.

There are several points to bear in mind about Fig. 3. First is that this disk model assumes a turbulent disk viscosity associated with a constant alpha viscous parameter $\alpha \sim$ a few $\times 10^{-3}$. Different values of $\alpha$, or perhaps more realistically a radial profile of $\alpha$ inspired from simulations of MHD turbulent disks, would result in different locations for regions of outward migration (Bitsch et al. 2014). Second is that this picture is timedependent: as the disk evolves, it loses mass and cools with the consequence that regions of outward migration progressively shift towards smaller orbital radii and smaller planet masses (Bitsch et al. 2015b).

\subsubsection{Some Recent Progress on Type I Migration}

The predicted rapid inward migration of planets of few Earth masses has stimulated much work on type I migration. A selection of recent works on type I migration is detailed below.

(i) Disk's magnetic field. The recent work by Guilet et al. (2013) has shown that there is an additional corotation torque on low-mass planets when the disk has a weak toroidal magnetic field. This new torque comes about because of advection-diffusion of the magnetic flux 
inside the planet's horseshoe region. The horseshoe trajectories of the gas cause an accumulation of the magnetic flux contained in the horseshoe region downstream of the U-turns. The magnetic pressure increases locally, and the thermal pressure decreases to maintain a mechanical equilibrium with the surrounding gas. This, in turn, decreases the local gas surface density if the disk's temperature is stationary. An azimuthal asymmetry of the horseshoe Uturns relative to the planet, which is related to the radial pressure gradient, makes this new corotation torque generally positive and favors outward migration. Its magnitude depends sensitively on the strength of the magnetic field and the magnetic resistivity (Guilet et al. 2013). We point out that the surface density perturbation obtained in the $2 \mathrm{D}$ laminar disk models of Guilet et al. (2013), where the effects of turbulence are modeled by a viscosity and a magnetic resistivity, is in excellent agreement with the time-averaged density perturbation found in the 3D MHD turbulent simulations of Baruteau et al. (2011a), which were for Saturn-mass planets in high aspect ratio disks (see the comparison in Fig. 3 of Baruteau et al. 2014). More work is needed in this area, in particular for more deeply embedded planets. When the disk has a strong toroidal magnetic field, horseshoe streamlines disappear and the corotation torque is replaced by a so-called MHD torque that is driven by angular momentum carried away by slow MHD waves excited in the planet's vicinity. The recent work by Uribe et al. (2015) shows that this MHD torque can be positive and also reverse type I migration, in agreement with earlier analytical and numerical works (Terquem 2003; Fromang et al. 2005).

(ii) Heating torque. Material accreted by a planet releases energy that heats up the disk gas in the planet's vicinity (located typically by a few Hill radii from the planet; the Hill radius is defined at Eq. 4). The gas flow near the planet implies that this released energy forms two hot lobes: (i) one in front of the planet in the azimuthal direction and inside the planet's orbit, and (ii) a second one behind the planet in the azimuthal direction and outside the planet's orbit (Benítez-Llambay et al. 2015). The gas density decreases at the location of the lobes to maintain a mechanical equilibrium with the surrounding gas. The under-dense lobe in front of the planet favors inward migration (its gravitational force on the planet has a negative azimuthal component), whereas the lobe behind the planet favors outward migration. The flow asymmetry near the planet, which is related again to the radial pressure gradient, makes the lobe behind the planet hotter and more under-dense. The resulting torque on the planet, termed "heating torque" by Benítez-Llambay et al. (2015), therefore favors outward migration. It is not a corotation torque since it is not associated with horseshoe U-turns. The magnitude of the heating torque depends sensitively on the planet's growth timescale and the disk opacity. The heating torque increases with the accretion rate and with increasing disk opacity (since higher opacities inhibit cooling). The results of BenítezLlambay et al. (2015) show that the heating torque can reverse type I migration of planets of 1 to 3 Earth masses that are located near $5 \mathrm{AU}$ if their mass doubling time is shorter than a few $\times 10^{4}$ years. Such short growth timescales could be obtained through pebble accretion.

(iii) Eccentricity. Despite the relative efficiency of the disk gas at damping eccentricities, a planet can maintain some level of eccentricity if it is forced by another planet, in particular if planets are in or near mean-motion resonances. Fendyke and Nelson (2014) have shown that the radial width of the planet's horseshoe region decreases with increasing planet eccentricity, and therefore so does the magnitude of the corotation torque. This clearly has important implications for the strength of type I migration in multi-planetary systems that contain Earth- to Neptune-mass planets.

(iv) Dynamical corotation torque. We have examined so far the various contributions to the type I migration torque for planets on fixed orbits, which assumes that the migration rate is directly given by the disk torque, and that the latter is independent of the migration 
rate. However, as a planet drifts in the disk, gas outside the horseshoe region eventually executes a unique horseshoe U-turn relative to the planet and thus contributes to the corotation torque. The corotation torque imparted by orbit-crossing gas scales with the mass flow rate across the orbit and therefore depends on the migration rate. This torque is negative if the planet moves inward, positive if it moves outward. It therefore causes a positive feedback on migration. It adds to the corotation torque due to the gas trapped inside the planet's horseshoe region, which has two contributions. First is the horseshoe drag caused by the gas in the horseshoe region executing U-turns relative to the planet, as in the case when there is no radial drift between the disk and the planet. Second is the torque the horseshoe region has to apply on the planet so that both migrate at the same rate. This torque, which we call the trapping torque, is perhaps more easily understood as the opposite of the torque the planet exerts on its horseshoe region to have it migrate at its own drift rate. The trapping torque is positive (negative) if the planet moves inward (outward); it thus yields a negative feedback on migration.

Adding all contributions together, we see that the corotation torque has in general two components: (i) the static corotation torque, which is the horseshoe drag, and (ii) the dynamical corotation torque, which is the sum of the trapping torque and the orbit-crossing torque (Masset and Papaloizou 2003; Paardekooper 2014). As this terminology implies, only the dynamical corotation torque depends on the migration rate and causes a feedback on migration.

The key quantity to assess whether feedback is negative or positive is a quantity called "vorticity-weighted coorbital mass deficit" (Masset and Papaloizou 2003). This quantity is well known for massive planets that open a gap around their orbit (see Sect. 3.2). Since low-mass planets do not or hardly deplete their horseshoe region, above quantity should be thought of as a vorticity or vortensity deficit, which comes about because orbit-crossing gas has a vortensity different from the averaged vortensity of the horseshoe region (Paardekooper 2014). For type I migrating planets, the vortensity deficit can be either negative or positive depending on the radial gradient of disk vortensity in isothermal disk models (where the gas temperature is kept stationary). Extension to nonisothermal, radiative disk models (including viscous heating, stellar irradiation and radiative cooling) has been carried out by Pierens (2015), where the vortensity deficit is primarily a surface density deficit brought about by radial variation of the gas entropy in the disk; the vortensity deficit can be positive or negative depending mostly on the radial gradient of disk entropy. Conditions for type I migration to runaway are described in Paardekooper (2014) for isothermal disks, and in (Pierens 2015) for non-isothermal disks. For this review, it is sufficient to take away that, in massive low-viscosity disks, the dynamical corotation torque can dramatically slow down inward type I migration (negative feedback), and possibly lead to runaway outward type I migration (case where positive feedback causes a runaway). In any case, the dynamical corotation torque can largely widen the range of orbital radii where outward migration can occur (Paardekooper 2014; Pierens 2015).

\subsection{Disk Migration of Massive Planets}

We provide in this section a short overview of planet-disk interactions for massive planets that open a gap around their orbit, their migration properties, and touch upon some aspects of their observable signatures. 


\subsubsection{Gap Opening}

The more massive a planet, the stronger its wakes, and the closer to the planet will wakes turn into shocks and deposit their energy and angular momentum. As the inner wake carries negative fluxes of energy and angular momentum, it will push gas inward, away from the planet's orbit. Similarly, the outer wake carries positive fluxes of energy and angular momentum and will push gas outward, away from the orbit. Massive planets thus progressively deplete their co-orbital horseshoe region, which becomes an annular gap. The final gap width is determined by the balance between the gap-opening gravity torque of the planet and the gap-closing viscous and pressure torques (Crida et al. 2006). In other words, the ability of a planet to carve a gap does not only depend on the planet's mass, or, actually, the planet-to-star mass ratio $\left(q=M_{\mathrm{p}} / M_{\star}\right)$. It also depends on the disk's aspect ratio, $h$, as $q / h^{3}$, and on the disk's turbulent alpha viscosity, $\alpha$, as $\alpha h^{2} / q$ (Lin and Papaloizou 1986; Crida et al. 2006).

We stress that there is no accurate definition or criterion as to when a planet opens a gap. The widely used gap-opening criterion formulated by (Crida et al. 2006) is for when the planet's co-orbital surface density drops to $10 \%$ its initial value. Of course this threshold value is arbitrary, and planets less massive than predicted by Crida et al's criterion can still open a partial gap or dip. This can be the case for planets down to few Earth masses in weakly turbulent disks (e.g., Rafikov 2002; Muto et al. 2010; Dong et al. 2011; Duffell and MacFadyen 2013; Duffell 2015). Also, this criterion is for planets on fixed orbits. The gap-opening criterion is modified by migration, particularly in massive disks, as recently highlighted by Malik et al. (2015). An illustration of this is the rapid inward migration expected for planets formed by disk fragmentation (e.g., Baruteau et al. 2011b), which is initially so rapid that planets have no time to carve a gap, unless or until significant gas accretion occurs (Zhu et al. 2012; Nayakshin and Cha 2013; Stamatellos 2015). We also point out the recent work by (Fung et al. 2014) who estimated via hydrodynamical simulations the gas surface density contrast inside and outside a planet gap. Note that simulations of MHD turbulent disks show that the width and depth of planet gaps can be somewhat different from viscous disk models (Papaloizou et al. 2004; Zhu et al. 2013).

Gap-opening planets are often invoked to explain the large cavities observed in transition disks (see, e.g., the review by Espaillat et al. 2014). It should be borne in mind that the width of a planetary gap varies with dust size. Dust grains up to few tens of microns are tied to the gas, so the width of their gap is just as narrow as in the gas. Dust grains in the $\mathrm{mm} / \mathrm{cm}$ range (depending on the local gas density) are generally not tied to the gas: they undergo significant radial drift and tend to concentrate in regions of pressure maxima (see Sect. 2.2.1). Simulations of planet-disk interactions show that the outer edge of a planet gap is generally a robust pressure maximum but the inner edge is not. This implies that $\mathrm{mm} / \mathrm{cm}$ grains initially beyond the planet's orbit can be trapped efficiently at the gap's outer edge, and those initially inside the planet's orbit will migrate inward (e.g., Zhu et al. 2012). A single massive planet could therefore simultaneously open a narrow gap in $\mu \mathrm{m}$-grains and a large cavity in mm-grains. We point out that large cavities in $\mu \mathrm{m}$-grains are not a natural expectation of planet-disk interactions, unless one invokes the presence of several massive planets in the disk (Zhu et al. 2011). Finally, we stress how uncertain it would be to apply predictions of gap widths and depths for the gas to observed gap structures in protoplanetary disks imaged in the $\mathrm{mm} / \mathrm{submm}$, like for example the (suggestive) gap structures seen in the HL Tau disk imaged by ALMA (ALMA Partnership et al. 2015).

Quite similarly, massive, yet unseen planets have been widely invoked to explain the spiral density waves observed in near-infrared images of protoplanetary disks. While spiral 
waves could be generated by other means, for example by gravitational instability, studies on the observability of the spiral wakes induced by a massive planet, coupling hydrodynamical simulations and radiative transfer calculations, are emerging (Juhász et al. 2015; Zhu et al. 2015). The work by Dong et al. (2015) provides convincing evidence that the two spirals seen in MWC 758 in polarized scattered light (Benisty et al. 2015) can be due indeed to an unseen outer planet companion of a few Jupiter masses.

\subsubsection{Formation of a Circumplanetary Disk}

Part of the gas that is progressively expelled from the planet's co-orbital region during the opening of a gap forms a circumplanetary disk. The recent discoveries of young, massive planets on wide orbits by direct imaging, like HD 100546 b (recently confirmed by Currie et al. 2014 and Quanz et al. 2015), has stimulated a number of recent works that have examined the structure, accretion rate and observability of circumplanetary disks. Accretion onto the protoplanetary disk is found to be inherently 3D and to proceed from high latitudes (e.g., Tanigawa et al. 2012; Szulágyi et al. 2014), can be stochastic and even launch outflows (Gressel et al. 2013). The observability of circumplanetary disks has been assessed by the prediction of distinct features in the gas kinematics (Perez et al. 2015) and spectral energy distribution (Zhu 2015; Eisner 2015; Montesinos et al. 2015).

\subsubsection{Type II Migration}

The migration of massive, gap-opening planets is driven by the wake torque and the corotation torque (both its static and dynamical components). The balance between both torques depends on how deep and wide the gap is, which, as we have seen above, depends on the planet's mass, the disk's aspect ratio and viscosity. For planets that open a deep gap around their orbit, which, roughly speaking, correspond to planets more massive than Jupiter, the corotation torque is largely suppressed and the wake torque is weakened. Still, the wake torque remains the main driver of migration. This corresponds to the so-called type II migration regime. It is directed inwards and runs on timescales longer than $10^{4-5}$ yrs. Recently, Dürmann and Kley (2015) have shown through numerical simulations that type II migration does not depend on the viscous inflow speed of the disk gas. This implies that type II migration is not tied to the disk's viscous evolution, as often thought, and that gas can cross the gap during the planet's migration. Type II migration is considerably slowed down when the planet mass becomes larger than the mass of the gas outside the planet gap.

\subsubsection{Type III Migration}

For planets that open a partial gap around their orbit, which is typically the case of Saturnmass planets, the corotation torque still plays a major role in the migration. At low to moderate gas surface densities at the planet's location, that is when the local Toomre parameter $Q \equiv c_{\mathrm{s}} \Omega_{\mathrm{K}} / \pi G \Sigma$ typically exceeds 10 , the migration regime is intermediate between the type I and type II regimes (e.g., Masset and Papaloizou 2003; Crida and Morbidelli 2007; D'Angelo and Lubow 2008). At larger gas surface densities at the planet's location ( $Q \lesssim 10$ ), the dynamical corotation torque becomes important and leads to a different migration regime termed type III migration (Masset and Papaloizou 2003). The co-orbital vortensity deficit featured by the dynamical corotation torque (see Sect. 3.1.4) becomes a surface 
density deficit or mass deficit (it is the mass that should be added to the planet's horseshoe region so that it gets the averaged surface density of the orbit-crossing gas). When the mass deficit becomes larger than the mass of the planet plus its circumplanetary disk, migration runs away, otherwise the dynamical corotation torque accelerates migration but does not cause a runaway (Masset and Papaloizou 2003). The planet's circumplanetary disk can have a significant impact on migration (D'Angelo et al. 2005; Pepliński et al. 2008; Crida et al. 2009).

\subsection{Disk Migration and the Observed Exoplanets}

This section discusses the role of planet-disk interactions in some of the observed properties of planetary systems. Sect. 3.3.1 deals with spin-orbit (mis-)alignments among hot Jupiters and their constraints on planet migration scenarios. Sect. 3.3.2 discusses the relevance of planet-disk interactions for the near- and non-resonant architecture of many of the multi-planet systems discovered by Kepler. For a detailed statistical comparison between the orbital properties of exoplanets and those predicted by global models of planetary formation and evolution, the reader is referred to, e.g., Benz et al. (2014) and Mordasini et al. (2015).

\subsubsection{Aligned and Misaligned Hot Jupiters: Constraints on Planet Migration Scenarios}

Observations of sky-projected planet obliquities have gained considerable importance in the past few years (the obliquity of a planet is the angle between the star's spin axis and the planet's orbital axis). Projected obliquities have been primarily obtained via the Rossiter MacLaughlin effect (e.g., Winn et al. 2005), which is a time-varying distortion in the spectral lines of the star caused by a transiting planet. Other techniques, like the analysis of starspots occultations by a planet, have also been used (e.g., Sanchis-Ojeda et al. 2011). Projected obliquities have been determined for a little more than 60 planets at the time of writing, the vast of majority of them are hot Jupiters with orbital periods below 10 days. Surprisingly, a fair number of hot Jupiters have a large projected obliquity (see Fig. 4). When their projected obliquity exceeds $90^{\circ}$, planets are said to be retrograde. It is seen that the projected obliquities seem to depend on the star's effective temperature, with hot Jupiters around hot stars being more likely misaligned (see, e.g., the review by Winn and Fabrycky 2015). These observations have considerable impact on evolutionary models of planetary systems, since they indicate that dynamical processes may commonly lead planets to acquiring large obliquities, which is often thought of as planets getting large inclinations at some point of their evolution. We mention that, under some circumstances, measuring the stellar inclination relative to the line of sight, $i_{\star}$, for example through asteroseismology, can constrain the true obliquity of a planet (a small $i_{\star}$ implying a large misalignment; Huber et al. 2013; Chaplin et al. 2013) and even determine it (Benomar et al. 2014). At the time of writing, true obliquities have been estimated for 11 planets, ${ }^{4}$ they exceed $30^{\circ}$ for 5 of them.

How to explain these observations? Hot Jupiters are thought to form at typically several AU from their central star, reaching orbital periods of a few days either by disk migration or by high-eccentricity migration. Disk migration is expected to preserve zero obliquity (e.g., Bitsch et al. 2013a) unless (i) the disk becomes misaligned with the stellar equator due to gravitational interactions with nearby stars (Bate et al. 2010; Batygin 2012; Picogna and Marzari 2015), or (ii) the star itself becomes misaligned, which might occur

\footnotetext{
${ }^{4}$ See, e.g., http://www.astro.keele.ac.uk/jkt/tepcat/rossiter.html.
} 


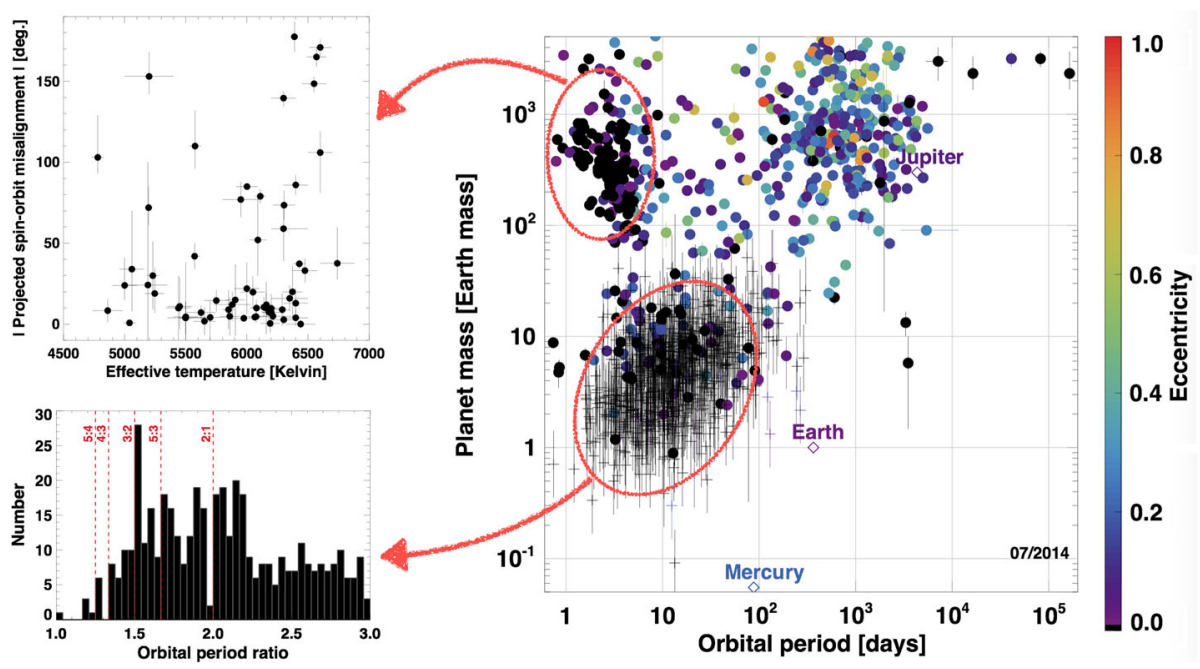

Fig. 4 Right: Mass, orbital period and eccentricity of observed exoplanets (filled circles), and for Mercury, Earth and Jupiter. Planets labeled by plus signs are planets detected by transit only, for which the mass is inferred from the mass-radius relationship $M \propto R^{2.06}$ for low-mass planets with both mass and radius determined. A $50 \%$ error bar on the mass has been added to these planets detected by transit only. Upper-left: projected obliquity versus stellar effective temperature. Lower-left: period ratio histogram for confirmed multi-planet systems, the period ratio of a few mean-motion resonances is shown by vertical dashed lines. All data were extracted from http://exoplanets.org

through star-planet tidal interactions (tidal flip of stellar axis, Cébron et al. 2013; Barker and Lithwick 2014). Note that the delivery of hot Jupiters by disk migration does not have to proceed solely by smooth type II migration. It may be assisted by the inward scattering of a giant planet resulting from interactions with one or more giant planet companions in the protoplanetary disk (e.g., Marzari et al. 2010). In the high-eccentricity migration scenario, a Jupiter-mass planet begins on an inclined, highly eccentric orbit that shrinks and circularizes due to tidal interactions with the central star. Mechanisms that may pump planet eccentricities and/or inclinations in the first place include (i) planet-planet scattering between two or more massive planets, a scenario that has been proposed to explain the broad distribution of exoplanet eccentricities (e.g., Rasio and Ford 1996; Chatterjee et al. 2008; Jurić and Tremaine 2008), and (ii) Kozai cycles with an inclined, probably stellar-mass companion (e.g., Wu and Murray 2003; Fabrycky and Tremaine 2007; Naoz et al. 2012). High-eccentricity migration can produce a broad range of planet obliquities depending on the excitation mechanism and the effectiveness of tidal interactions at damping obliquities (which depends on the star's mass, rotation period, the planet's mass and orbital separation etc.).

Interestingly, it has been suggested that perhaps all hot Jupiters formed by high eccentricity migration with a broad range of initial obliquities, and that tidal interactions with the central stars were more or less efficient at reducing obliquities over the age of the systems (that is, at aligning the stellar spin axis with the planet's orbital axis, Triaud et al. 2010; Albrecht et al. 2012). (Albrecht et al. 2012) argued that aligned systems would be those where the timescale for spin-orbit alignment is shorter than the age of the system. They used the estimates of (Zahn 1977) for the tidal synchronization timescales in close binary stars, showing that tidal alignment should be more efficient for cooler stars that harbor a convective envelope. While Zahn's theory does predict an increase in 
the alignment timescale with the star's effective temperature, in practice it gives alignment timescales that are at least one or two orders of magnitude longer than the age of the systems where projected obliquities have been measured (Albrecht et al. 2012; Ogilvie 2014). This shows the limitations in applying Zahn's theory of tidal evolution of binary stars to hot Jupiters. This result has nevertheless stimulated much work on star-planet tidal interactions. In particular, the conditions for tides to produce alignment faster than orbital decay lead to the prediction that there should be as many retrograde aligned planets as prograde aligned ones, which is not what is observed (Lai 2012; Rogers and Lin 2013).

Could measurements of projected obliquities help distinguish and/or constrain the mechanisms of hot Jupiter migration? Answer to this important question probably requires a statistical comparison between model predictions and observations. We mention the recent work by Crida and Batygin (2014), who compiled the predictions of high-eccentricity migration models in terms of projected obliquities, and compared them with observations. They find that the models successfully reproduce the distribution of projected obliquities beyond $40^{\circ}$, but they all underestimate the proportion of systems with low obliquities. An alternative scenario mentioned above is the possibility that the disk can be misaligned relative to the star's equator due to the interaction with a binary companion star. Crida and Batygin (2014) use Monte-Carlo simulations to predict the distribution of projected obliquities according to this scenario. They find that, (i) just like for the combined high-eccentricity and tidal friction scenario, the misaligned disk migration scenario can robustly reproduce the distribution of projected obliquities beyond $40^{\circ}$, but (ii) only the aligned disk migration scenario can account for the number of aligned and nearly aligned hot Jupiters, depending on the semi-major axis distribution of the binary companions. Inclusion of magnetic interactions between the disk and the central star allows the misaligned disk migration scenario to reproduce the observed trend between misalignment and stellar mass (Spalding and Batygin 2015). These comparisons highlight that (i) current observations cannot disentangle between the high-eccentricity migration scenario and the disk migration scenario in a misaligned disk to explain misaligned hot Jupiters, and that (ii) delivery of aligned hot Jupiters via disk migration is necessary to account for the number of aligned hot Jupiters. Chemical depletions (e.g., $\mathrm{O}$ and $\mathrm{C}$ abundances) in the atmosphere of hot Jupiters could be a way forward in constraining their migration scenario (Madhusudhan et al. 2014). We also point out that the fraction of observed aligned hot Jupiters does not seem to vary significantly with orbital period $^{5}$ which, in our opinion, is a challenge to the above tidal alignment theory. We finally mention the recent work by Mazeh et al. (2015), who show that the trend of lower obliquity around cooler stars extends to Kepler objects of Interest with orbital periods up to at least 50 days, for which star-planet tidal re-alignment should be largely negligible.

\subsubsection{Architecture of Kepler's Multi-Planetary Systems}

Kepler has uncovered a large population of Earth- to Neptune-mass planets in compact multi-planetary systems with orbital periods between 1 and 100 days. The distribution of period ratios between all pairs of planets of a given system is displayed in the lower-left corner of Fig. 4 for Kepler's confirmed multi-planet systems. There are two salient points.

\footnotetext{
${ }^{5}$ This is inferred from the sample of planets listed in http://exoplanets.org that have a minimum mass greater than $0.5 M_{\mathrm{J}}$. Defining as aligned a planet with projected obliquity less than about $40^{\circ}$, we find that, in the range of orbital periods between 1 and 3 days, there are 18 aligned and 7 misaligned planets, while for orbital periods between 3 and 5 days, there are 16 aligned and 8 misaligned planets.
} 
First, there are many planet pairs far from resonances, while the usual expectation from disk migration models is that Kepler-mass planets should form resonant planet pairs, that is, one would a priori expect only spikes in this distribution at the location of the resonant period ratios, like $2: 1,3: 2$ etc. Second, planet pairs near resonances tend to have period ratios somewhat greater than resonant. It is worth noting that, despite a much smaller number statistics, a similar trend can be seen for planetary systems detected by RV techniques only. We point out that some features in the period ratio distribution may be artifacts of unseen additional companions (Steffen 2013). For example, a two-planet system with a period ratio $\sim 1.9$ could actually be a three-planet resonant system where the middle planet, missed by the transit observation, is in $4: 5$ mean-motion resonance with the innermost planet, and in $3: 2$ mean-motion resonance with the outermost planet.

How to explain these observed features? One model that has been recently put forward is in-situ growth. Here, it is important to stress that this is not in-situ growth all the way from micrometer-sized grains to planetary-sized objects, but in-situ growth of pre-formed and pre-evolved Mars- to Earth-sized embryos in a gas-free disk, and that these embryos may well have been delivered previously by disk migration, before the protoplanetary disk gets cleared out. The period ratio distribution predicted by the in-situ growth model of Hansen and Murray (2013) tends to produce too few close and resonant planet pairs compared to observations. The recent work by Ogihara et al. (2015) shows that planet embryos actually form very rapidly in the inner parts of protoplanetary disks, and that therefore disk migration cannot be neglected. Inclusion of disk migration in the "in-situ" growth calculations of Ogihara et al. (2015) shows that disk migration leads this time to an excess of close pairs of planets compared to observations.

Another model that has been highlighted is that a resonant planet pair formed by disk migration may evolve away from resonance due to star-planet tidal interactions, which tend to slowly increase the planets' period ratio over time, at a rate that depends on the star-planet separation (Papaloizou 2011; Lithwick and Wu 2012). While tides may indeed account for some planet pairs being just wide of resonance, we note that a similar trend is observed for planet pairs above 10 days, for which tides should be inefficient (Baruteau and Papaloizou 2013).

More recently, disk migration of planet pairs has been revisited. Baruteau and Papaloizou (2013) have shown that, at the typical location of Kepler planets, the wakes of the planets should be shock waves, which implies that: (i) the planets should open partial gaps around their orbits, and (ii) the wakes deposit energy and angular momentum in the co-orbital region of each planet, with the consequence that the planets tend to repel each other over time. This stresses that disk migration models do not necessarily predict Kepler-mass planets to form resonant pairs. Turbulence in the disk is another way to explain why many low-mass multiple planet systems are near- or non-resonant (Pierens et al. 2011; Rein 2012), and may play a prominent role in the formation of closely packed systems (Paardekooper et al. 2013).

\section{Internal Evolution of Planets}

We now turn our attention to individual planets, treating them no more just as point-like masses, but as geophysical objects that are characterized by a radius, a luminosity, an atmospheric and a bulk compositions, which can all evolve in time.

A small fraction of self-luminous objects like $\beta$ Pic b (Lagrange et al. 2009) among the known extrasolar planets has been directly detected at young ages, using coronagraphs and dedicated data reduction procedures, and a handful of objects has even been observed 
during their formation, which occurs on a timescale of $\sim 10^{7}$ years (e.g., Quanz et al. 2015). However, the large majority of known exoplanets orbits stars that are at least $\sim 100$ Myrs old with a typical age of several Gyrs, meaning that we usually observe (exo)planets at an epoch much after their formation. Many of these objects are then too faint or too close to their star to be detected directly. More often their mass can, however, be inferred from radial velocity measurements. Further, if the planets are transiting their star, it becomes possible to measure their radius if the radius of the host star is known. Transits also allow to probe the planetary atmospheres and to get hints at the planet's abundances and day-side emission. The combination of radial velocity and transit measurements finally yields the mean density of a planet, which is its first rough but important geophysical characterization (e.g., Dressing et al. 2015; Santos et al. 2015).

The number of planets for which various observational data is available has increased enormously in the past two decades (e.g., Mayor et al. 2011; Borucki et al. 2011b; Marcy et al. 2014). One of the major goals of the research on (exo)planets is to better understand the physics of planet formation thanks to this data, including in particular the Solar system. A typical approach is to compare the results from theoretical planet formation models and the observational constraints from the aforementioned techniques. However, in order to draw conclusions about the formation of planets from properties that we observe today like orbital parameters, mass, radius, luminosity and composition, one needs to understand not only how planets form, but also how they evolve from formation until the epoch of observation. The reason for this is that the evolution spans Gyr-long timescales, and the link between observation and formation can be non-trivial and potentially mask the original imprints of formation.

Close-in low-mass planets are an illustrative example. Their formation mechanism is currently debated (see, e.g., Sect. 3.3.2). Additional constraints that can be derived from their geophysical characteristics like for instance the $\mathrm{H} / \mathrm{He}$ envelope mass are therefore very valuable to distinguish different formation scenarios (Sect. 4.5). However, because of envelope evaporation (atmospheric escape), some planets might have started with a gaseous envelope after formation but then lost it during the evolutionary phase.

Directly imaged planets are another example. Even for these young planets, which are typically only a few Myrs old, the question of understanding their temporal evolution since formation is of central importance when it comes to infer their mass based on their luminosity. This mass-luminosity relationship is in turn of high interest for formation theories as will be discussed in Sect. 4.2: it constrains the physics of gas accretion and potentially even the mode of formation, namely core accretion (Perri and Cameron 1974; Mizuno 1980; Bodenheimer and Pollack 1986) or gravitational instability (Toomre 1981; Boss 1997; Durisen et al. 2007). Not only must evolutionary models be well-suited to describe the governing physics, but also the correct initial conditions for the planet's evolution must be known, meaning that formation and evolution must be linked self-consistently.

In this section, we mostly concentrate on how to derive constraints on formation models from studying the long-term internal (mainly thermodynamical) evolution of planets after the dissipation of the protoplanetary disk, which is the link between formation and observation. Concentrating mainly on planets with primordial $\mathrm{H} / \mathrm{He}$, we address some of the most important aspects of planetary evolution like cooling and contraction, the mass-luminosity relation, and the bulk composition as expressed in the mass-radius and mass-mean density relations. We also quickly touch upon other topics that can play important roles like atmospheric escape, radius inflation and deuterium burning. For further aspects of the theory of planetary evolution, including crucial elements like high-pressure physics and equations of state, the internal rotation of planets and their shape, the specific properties and cooling histories of the Solar system planets, or potential phase separations of elements in planetary 
interiors (to name just a few), the reader is referred to the reviews of Stevenson (1982); Guillot (2005); Fortney and Nettelmann (2010); Chabrier et al. (2014); Guillot and Gautier (2014) or Baraffe et al. (2014).

\subsection{Thermodynamical Evolution: Cooling and Contraction}

The thermodynamical evolution of giant planets after formation at constant mass is controlled by cooling and contraction. They evolve from a comparatively hot post-formation state characterized by high entropy, high luminosity, low degeneracy, and large radius to a colder state with low entropy, low luminosity, higher degeneracy, and smaller radius (e.g., Guillot 2005). In contrast to stars, due to their comparatively low internal temperature, giant planets do not radiate away energy released by thermonuclear fusion as they are not massive enough to allow for fusion in their interior. The only exception may be a short stage of deuterium burning in planets with masses in excess of $12-13 M_{\mathrm{J}}$, as we will see in Sect. 4.3. But, for the bulk of giant planets, cooling and contraction are the main source of internal luminosity (Hubbard 1980), which can be seen from the fact that Jupiter, Saturn and Neptune emit more energy than they receive from the Sun.

The total amount of energy that can be radiated away by a giant planet during its lifetime is primarily determined by its post-formation entropy (Sect. 4.2). For terrestrial planets, such as Earth, another important contributor to the internal luminosity, besides delayed secular cooling, is the radioactive decay of unstable heavy element isotopes (e.g., Urey 1955; Hofmeister and Criss 2005). For the Earth, the total intrinsic heat flux is around 47 TW (which is only about $0.03 \%$ the solar irradiation flux), of which about half is estimated to arise from radiogenic heating (Kamland Collaboration et al. 2011). For Jupiter, the intrinsic luminosity (which is of the same order as the solar irradiation flux, Guillot and Gautier 2014) is largely dominated by the cooling and contraction of the fluid $\mathrm{H} / \mathrm{He}$ envelope; a possible solid core could contribute a few percent, while the radiogenic contribution is tiny $\left(\sim 10^{-4}\right)$.

The cooling of gas giant planets is caused by the radiation escaping through their atmosphere, which decreases the total energy of the planet. Because of virial equilibrium, for self-gravitating objects composed mainly of ideal gas, radiative escape causes somewhat counter-intuitively the interior to heat up (e.g., Guillot 2005). Such objects are therefore said to have a negative heat capacity: the loss of energy at their surface leads to an increase in their internal energy and central temperature, which is compensated for by the object's contraction. This regime applies to low-mass pre-main-sequence stars, as well as extremely young hot giant planets at ages less than $10^{5-6}$ years they are not yet significantly degenerate (Graboske et al. 1975).

The situation is quite different for older giant planets however, for which cooling and contraction cause the interior temperature to decrease. This is because the thermal pressure needed to balance the planet's gravity is established by degenerate electrons, which increase their mean kinetic energy, in contrast to ions, which decrease their kinetic energy and cool, yielding most of the intrinsic luminosity (Guillot 2005). In this phase, giant planets behave qualitatively like degenerate brown dwarfs (Graboske et al. 1975).

The time evolution of the cooling of a planet, and thus its internal evolution, depend on how energy is transported from the interior to the top and then through the atmosphere of the planet. The atmosphere can be thought of as a blanket which, with increasing thickness (i.e., optical depth), diminishes the planet's ability to cool, making it stay hot and have a larger physical radius for a longer period of time at high opacity (Burrows et al. 2007). For strongly irradiated planets the cooling timescale also increases: the incoming radiation makes the atmospheres more isothermal, reducing the effectiveness of radiative energy transport, as 

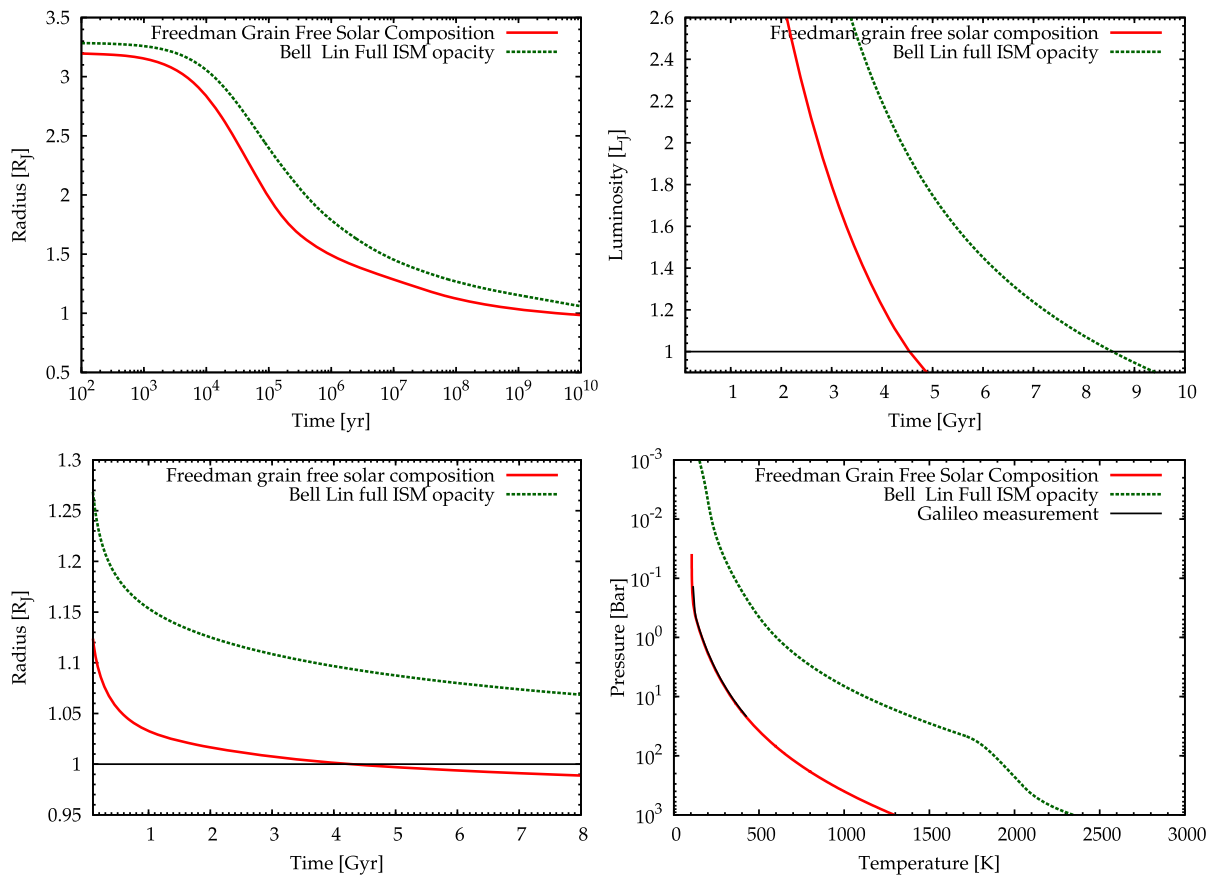

Fig. 5 Calculation of the internal evolution of a Jupiter-mass planet for two different input opacities: full ISM grain opacities (Bell and Lin 1994, green dotted curves), and Solar abundance molecular opacities (Freedman et al. 2008, red solid curves). The time evolution of the planet's $\tau=2 / 3$ radius is shown in the upper-and lower-left panels with two different time windows. The time evolution of the planet's luminosity is in the upper-right panel, and the pressure-temperature profiles of the planet's atmosphere at Jupiter's current age is displayed in the lower-right panel. The black solid curve in the lower-right panel shows the pressure-temperature profile measured by the Galileo probe (Seiff et al. 1996)

the atmospheric temperature gradient decreases (Burrows et al. 2000; Guillot and Showman 2002).

An example of planetary evolution calculation illustrating the impact of opacities is shown in Fig. 5 (Mordasini et al. 2012a). For such calculations, the fundamental equations for a planet's internal structure (equations of mass conservation, hydrostatic equilibrium, energy conservation, and energy transport by radiation or convection) are solved (e.g., Guillot and Gautier 2014) which allows the construction of evolutionary sequences based on the principle that the luminosity is equal to minus the temporal change of the planet's total energy. The post-formation evolution of a Jupiter-like planet is simulated assuming (ad hoc) either very high opacities caused by ISM grains, or more realistic grain-free molecular opacities for a solar composition gas which are about three orders of magnitude lower (see Bell and Lin 1994 and Freedman et al. 2008 for the grain and molecular opacities, respectively). For both sets of opacities, the planet has the same initial entropy. Note that planets with the same set of opacities, but different initial entropies, should still get the same structure in the end because the initial evolutionary timescales are short, at least if the post-formation entropies are not very low (Sect. 4.2). Figure 5 displays the time evolution of the planet's radius and luminosity, as well as the pressure-temperature profiles of the planet's atmosphere at Jupiter's current age. One clearly sees that for ISM grain opacities (in reality the grains would fall out of the atmosphere on a very short timescale, Mordasini 2014), the planet's 
radius remains larger than for molecular opacities. Also, after a few Gyrs, the planet luminosity obtained with ISM grain opacities is about a factor 2 larger than with molecular opacities. This is expected, as with ISM opacities the planet cools much more slowly, so that at late evolutionary times the planet has retained more heat than if it had cooled down with low molecular opacities. Only molecular opacities can reproduce Jupiter's present day radius, luminosity, and the pressure-temperature profiles measured by the Galileo probe (Seiff et al. 1996).

Energy transport in the interior of gaseous planets is commonly assumed to be due to convection. This is because the interior is usually characterized by high opacities, such that Schwarzschild's criterion for convection is satisfied (but see also Guillot et al. 1995). However, different means of energy transport might make planetary interiors considerably deviate from the picture of efficient convective energy transport. If planetary envelopes have a compositional gradient with mean molecular weight increasing towards the interior, the large-scale energy transport by convection could be shut down. The reason for this is that blobs of gas perturbed to upper layers of the atmosphere must sink back downward because the surrounding material has a lower density due to a smaller mean molecular weight. The possible implications for planetary structures were discussed by Stevenson (1985) and recently revisited by Leconte and Chabrier (2012). Following these studies, layers which are unstable according to Schwarzschild's criterion, but stable when including the compositional gradient, are thought to become semi-convective, exhibiting many convective layers separated by thin conductive layers with steep compositional gradients. The conductive energy transport is established by the thermal motion of electrons and ions. Planets with such semi-convective envelopes are thought to cool much slower, as the conductive boundary layers hamper efficient energy transport. It has been suggested that this phenomenon could be responsible for Saturn's high luminosity (Leconte and Chabrier 2013), which is too high when compared to classical, fully convective models (Pollack et al. 1977). Semi-convection is thus an alternative to the classical explanation that Saturn's excess luminosity is caused by the phase separation of hydrogen and helium (He insolubility) and a subsequent helium "rain" into the deeper layers, which releases gravitational potential energy (e.g., Stevenson and Salpeter 1977).

There are two important processes to assess the extent to which strong compositional gradients, and therefore semi-convection, are present in planetary envelopes. First, the solid core present in planets thought to have formed by core accretion might dissolve, leading to an increasing mean molecular weight in the layers close to the core-envelope boundary. This has been studied by Guillot et al. (2004) and Wilson and Militzer (2012), who found that a dissolution and subsequent mixing of the core might be possible. Second, during the planet formation phase, the destruction and heavy element deposition by planetesimals breaking up in the envelope similar to comet Shoemaker-Levy 9 could also lead to compositional variations. The ablated material could either stay suspended near the location where it has been deposited, settle towards deeper regions, or get mixed through the envelope by convection (Mordasini et al. 2006; Iaroslavitz and Podolak 2007). Clearly, this can lead to compositional gradients in the planet's envelope that are determined by the formation process. In this way the planetesimal accretion history might influence the planet's evolution even at late times.

\subsection{Initial Conditions for Planetary Evolution}

The early evolution of gaseous planets depends crucially on the amount of heat retained from the formation process. Depending on whether they form with a low, average or high initial 
Fig. 6 Summary of the various pathways to giant planet formation leading to different post-formation entropies (Mordasini et al. 2012a). The gas surface density plot in the upper-right corner illustrating gravitational instability is taken from Boley (2009). $M_{\text {ini }}$ denotes the initial mass of the clump formed by gravitational instability. Note that several pathways have to date not been simulated quantitatively

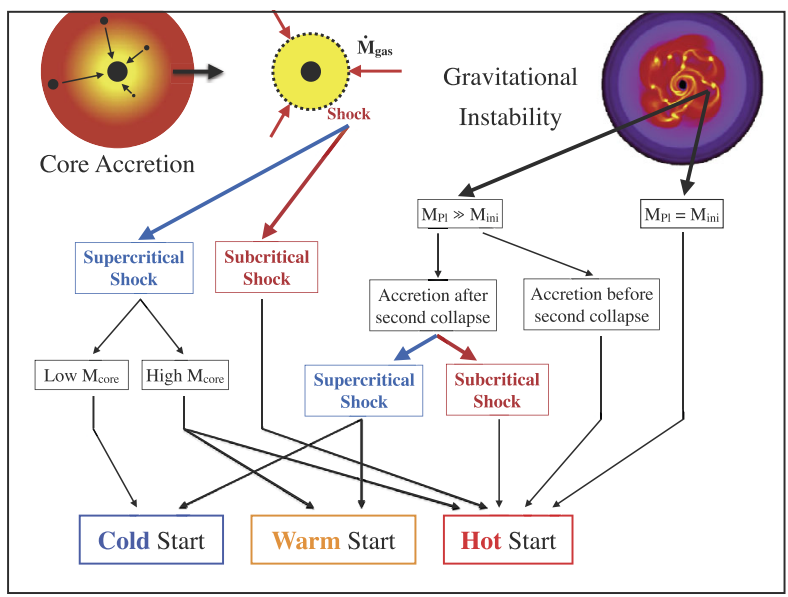

entropy (with a correspondingly low, average and high luminosity, radius and temperature), planets are said to have "cold", "warm" or "hot" start initial conditions. A typical cold start post-formation entropy is $\sim 8-9 k_{\mathrm{B}}$ /baryon ( $k_{\mathrm{B}}$ denotes the Boltzmann constant) whereas a typical hot start post-formation entropy is $\sim 11-15 k_{\mathrm{B}}$ /baryon (see, e.g., Marley et al. 2007; Spiegel et al. 2011; Mordasini 2013; Marleau and Cumming 2014). Given the significant impact that such initial conditions can have on the early internal evolution of giant planets, we first review the expected range of initial entropies for the two mainstream scenarios of giant planet formation: core accretion and disk gravitational instability. This is also summarized in Fig. 6, but it should be noted that several assumptions have not been validated yet with quantitative simulations, especially in the gravitational instability scenario.

(i) Core accretion. Traditionally, planet formation via core accretion is thought to produce gaseous planets with a low initial entropy (Fortney et al. 2005; Marley et al. 2007). The reason for this is the assumption that the accretion shock produced on the planet's surface by the infalling gas is radiatively efficient (or "super-critical"). For such a shock structure (found, e.g., for gas accretion on the first stellar core, Commerçon et al. 2011), the gravitational potential energy released at the shock is fully radiated away and none of it is incorporated in the convective interior of the planet. By analogy with the stellar case (e.g., Hartmann et al. 1997), planets undergoing such "cold" accretion will start their evolution with a low initial entropy and low internal temperature, dubbed "cold start".

However, the assumption that the shock is radiatively efficient is quite uncertain. If, on the contrary, the accretion shock is radiatively inefficient (or "sub-critical"), that is if the energy released at the shock is not radiated away (as found for gas accretion on the second stellar core, see Vaytet et al. 2013), planets will have high post-formation entropies, actually similar to those assumed in classical purely evolutionary simulations with a hot start (Mordasini et al. 2012a). The intermediate case where the accretion shock is neither radiatively efficient nor inefficient will lead to warm starts (Spiegel and Burrows 2012). Furthermore, and somewhat surprisingly, the formation of a massive solid core increases the post-formation entropy (Mordasini 2013; Bodenheimer et al. 2013). The requirement for this is an increased solid surface density $\left(\Sigma_{\mathrm{s}}\right)$ in the protoplanetary disk, which results in a higher solid accretion luminosity within the planet and a higher final core mass. Planets forming in disks with a high $\Sigma_{\mathrm{s}}$ will go into runaway gas accretion earlier, as the core also forms more rapidly. Therefore, gas is accreted onto a protoplanet with a larger radius, as the planet has had less time 
to cool and is more strongly stabilized against contraction owing to the increased planetesimal accretion luminosity. A larger radius means a weaker shock, therefore less gravitational potential energy released by the gas before it is accreted onto the planet. The gas is then incorporated into the planet at a larger entropy, also leading to warm starts (Mordasini 2013; Bodenheimer et al. 2013).

(ii) Disk gravitational instability. Another pathway to the formation of gaseous planets is gravitational instability (GI) in the outer parts of protoplanetary disks (typically beyond 50 to $100 \mathrm{AU}$; see, e.g., Boley et al. 2010). The possibility that GI occurs, the location where fragmentation sets in, and the subsequent internal and orbital evolutions of the clumps depend on the disk's mass, its structure and, critically, its cooling time scale (see, e.g., the reviews by Helled et al. 2014 and Kratter and Lodato 2016). Analytical arguments based on the Toomre (Toomre 1981) and cooling criteria (Gammie 2001) show that at smaller distances from the star, in a disk that is dense enough to be gravitationally unstable, it is not possible to cool rapidly enough to fragment, so that the two criteria cannot be simultaneously fulfilled (Rafikov 2005). In the outer parts of the disk, gravitational instability could instead occur early in the disk's evolution when it is still massive and accreting rapidly from the protostellar cloud (D'Angelo et al. 2011). But it is then unclear if bound clumps forming at this stage survive migration, evolve into planets, massive brown dwarfs or stellar companions (e.g., Stamatellos and Whitworth 2009), or if they get tidally disrupted (e.g., Nayakshin 2010; Forgan and Rice 2013).

Because in the case of a gravitational collapse the material is thought to not process through a shock, GI should yield hot starts. This has been found indeed in the numerical simulations of Galvagni et al. (2012), who report an entropy of $\sim 15 k_{\mathrm{B}} /$ baryon. However, if the collapsed protoplanet continues to accrete gas after it has fully collapsed (called "second collapse", analogous to stellar formation, a second collapse denotes the collapse after the molecular hydrogen has been dissociated) an accretion shock can potentially develop. The more mass is added through a super-critical shock onto the initial GI unstable object, the lower the final post-formation entropy of the planet (Mordasini et al. 2012a). If, however, the initially unstable object accretes its mass before the second collapse, it should rather have a hot start as the large radius of the clump should prevent the formation of strong shocks.

We point out that in "classical" planetary evolution calculations, which do not treat the formation phase, it is often simply assumed that planets start with an arbitrarily high initial entropy (that is, a hot start; see, e.g., Burrows et al. 1997; Baraffe et al. 2003). Because the Kelvin-Helmholtz (KH) timescale is short for large entropies, the initial condition is quickly erased such that after $\sim 10^{7}-10^{9}$ years for 1 and $10 M_{\mathrm{J}}$ planets, respectively, the luminosities in the cold and hot start cases converge to the same evolutionary tracks (see, e.g., Marley et al. 2007; Spiegel et al. 2011; Marleau and Cumming 2014). Therefore, the choice of an arbitrarily high initial entropy does not affect predictions on the planet appearance at ages $>10^{7}-10^{9}$ years. But it will do so for younger planets. This is particularly important for directly imaged planets in young stellar systems, for which the deduction of the planet's mass based on the planet's luminosity depends on the underlying assumptions of the utilized planetary evolutionary tracks.

A comparison between hot and cold start calculations is illustrated in Fig. 7 (updated from Marleau and Cumming (2014)). It shows the time evolution of the luminosity of planets with a mass of 1, 2, 3, 5, 10, 15, 20 and $25 M_{\mathrm{J}}$ for cold (blue curves) and hot initial conditions (red curves). Observational data of directly imaged planets are overplotted (updated from Neuhäuser and Schmidt 2012). One can see that for the specific evolutionary tracks shown in the figure, cold and hot start tracks converge after $\sim 10^{8}$ years. The mass degeneracy for a given age and luminosity is obvious when looking at the figure: at an age of $\sim 4$ Myrs, a 
Fig. 7 Luminosity as a function of time from theoretical models (Marleau and Cumming 2014) compared with directly imaged objects with a hot-start mass below 25 Jovian masses (data compiled by Neuhäuser and Schmidt 2012). The lines show cooling curves for $M=1,2,3,5$, $10,15,20$ and $25 M_{\mathrm{J}}$ (bottom to top) assuming a cold start with a post-formation entropy of $9.5 k_{\mathrm{B}}$ /baryon (blue lines) or a hot start (red lines). Figure updated from Marleau and Cumming (2014)

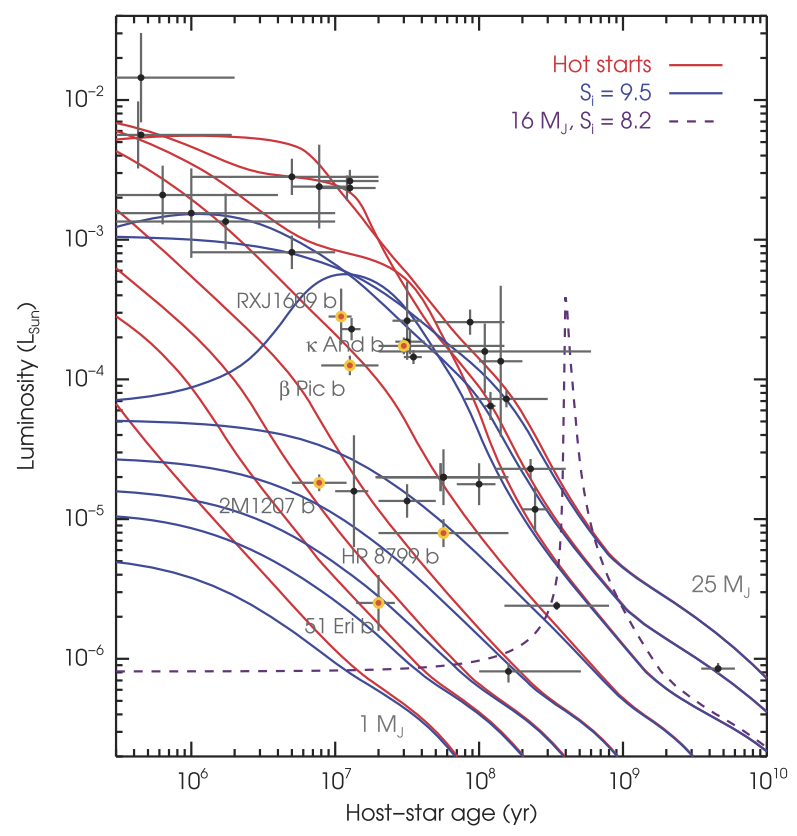

cold-start planet of $10 M_{\mathrm{J}}$ and a hot-start planet of $3 M_{\mathrm{J}}$ have the same luminosity. For cooler starts than assumed in the figure, the time after which the evolutionary tracks of cold and hot starts converge becomes longer and the difference in masses for a given age and luminosity increases.

\subsection{Deuterium Burning in Massive Planets}

Traditionally, gaseous objects which are able to fuse deuterium, but which are not massive enough to burn hydrogen on a stable main sequence, are called "brown dwarfs" (see, e.g., Boss 2007). They were first theorized to exist in the 1960s (Kumar 1963; Hayashi and Nakano 1963). The distinction between massive gaseous planets and brown dwarfs has been based on the hypothesized formation mode, with formation in a protoplanetary disk for giant planets, and gravoturbulent fragmentation of a molecular clump (i.e., a star-like formation mode) for brown dwarfs (Chabrier et al. 2014). In principle, it is well possible that, in massive protoplanetary disks, planets form with masses in excess of 13 Jupiter masses via core accretion (Mordasini et al. 2009b), and it has been shown that such planets are able to burn deuterium in the layers on top of their solid core (Baraffe et al. 2008).

Classical studies in the brown dwarf regime include Burrows and Liebert (1993); Saumon et al. (1996); Chabrier and Baraffe (2000); Burrows et al. (2001); Baraffe et al. (2003). They put a mass limit for the onset of deuterium burning at roughly $13 M_{\mathrm{J}}$. Deuterium burning in forming planets that harbor a solid core has been studied systematically in Mollière and Mordasini (2012) and Bodenheimer et al. (2013), and the mass threshold for deuterium burning is found to be $\sim 13 M_{\mathrm{J}}$ as well. For planets, the exact threshold value can vary with the helium content, the metallicity and deuterium fraction (Spiegel et al. 2011; Mollière and Mordasini 2012). It also varies with the core mass (Mollière and Mordasini 2012; 
Bodenheimer et al. 2013), since a more massive core leads to a higher post-formation entropy (see Sect. 4.2) and therefore a higher internal temperature, which increases the deuterium burning rate (Mordasini 2013; Bodenheimer et al. 2013).

In general, deuterium burning proceeds via the reaction

$$
\mathrm{H}+\mathrm{D} \rightarrow{ }^{3} \mathrm{He}+\gamma,
$$

with an energy release of $5.494 \mathrm{MeV}$ (Fowler et al. 1967). Since the hydrogen and deuterium ions need to be brought close enough to overcome the Coulomb repulsion and fuse, the reaction rate depends very strongly on the temperature. It is limited by the fact that, at the central temperatures in the objects of interest, only a small fraction of ions have kinetic energies large enough to tunnel through the ions' repulsive Coulomb barrier. Screening plays an important role too, as the electrons that are present in the fully ionized interiors of the planet effectively shield the ions from each other during their approach, decreasing the kinetic energy needed for the tunneling probability. As the objects are already considerably degenerate in their interior, classical Debye-Hückel shielding is not sufficient for these objects and screening theories for degenerate material need to be invoked (see, e.g., Dewitt et al. 1973; Graboske et al. 1973).

Deuterium burning can have a very strong dependence on temperature. The deuterium burning rate $\left(\epsilon_{\mathrm{D}}\right.$, energy per unit mass and time) can be expressed as a power-law function of temperature $T$ (see, e.g., Kippenhahn and Weigert 1990):

$$
\epsilon_{\mathrm{D}} \propto \rho X_{\mathrm{D}} X_{\mathrm{H}} T^{v}
$$

with $\rho$ the gas density, $X_{\mathrm{H}}$ and $X_{\mathrm{D}}$ the mass fractions of hydrogen and deuterium, respectively, and

$$
v=\frac{80}{3}\left(\frac{10^{5} \mathrm{~K}}{T}\right)^{1 / 3}-\frac{2}{3} .
$$

The power-law exponent of the temperature thus depends itself on the temperature. For deuterium burning in low-mass protostars, it is often assumed that $v=11.8$ (Stahler and Palla 2005), which corresponds to temperatures of $\sim 10^{6} \mathrm{~K}$. The coolest deuterium burning objects (with masses of $\sim 13 M_{\mathrm{J}}$ ) burn deuterium at central temperatures of roughly $4 \times$ $10^{5} \mathrm{~K}$ (see, e.g., Mollière and Mordasini 2012), corresponding to $v \sim 16$. Note, however, that electron screening in these objects may somewhat decrease $v$ (Kippenhahn and Weigert 1990).

The outcome of deuterium burning is quite different whether a hot start or a cold start is assumed. Hot start objects, which might be rather related to "classical" brown dwarfs, start their evolution with a large radius and a high temperature (see Sect. 4.2), and then contract slowly with time, starting the deuterium burning process once their central density is high enough. The main effect of deuterium burning is to slow down the contraction. Cold start objects, however, have quite low post-formation entropies, resulting in small radii and small internal temperatures (see Sect. 4.2). The fact that their internal temperature is smaller than in hot start objects, while having smaller radii, is due to the partial degeneracy of their interior. When cold start planets reach internal temperatures that are high enough for the onset of deuterium burning, they have much higher densities than hot start objects. The effect of deuterium burning for cold start planets is not a decreased contraction speed, but a re-inflation to larger radii. The increase in planetary luminosity for these objects therefore does not exclusively stem from the deuterium burning itself, but also from the later recontraction of the planets inflated by deuterium burning. 
Fig. 8 Luminosities of cold and hot start objects with a mass of 5 , 10, 15, 20, 25 and 30M (from bottom to top). The red solid curves show evolutionary calculations with a high initial entropy (hot start), taken from Burrows et al. (1997). The black solid curves show combined formation and evolutionary calculations of objects formed by core accretion with a low post-formation entropy (cold accretion). The high luminosities at around $1 \mathrm{Myr}$ are due to the gas accretion shock, they rapidly fall as gas accretion is (artificially) terminated (Mollière and Mordasini 2012)

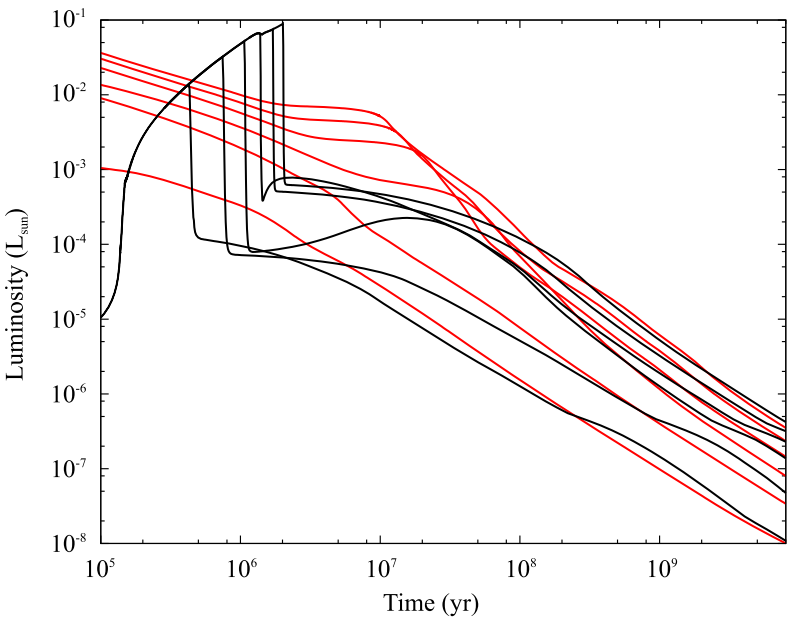

In general, the deuterium number fraction in the hydrogen and helium dominated atmospheres is quite low, $N_{\mathrm{D}} / N_{\mathrm{H}} \sim 2 \times 10^{-5}$ (Prodanović et al. 2010). Therefore, the phase of deuterium burning is rather short, between 1 and 100 Myrs, depending on the mass and formation mode (cold/hot).

Illustrative luminosity tracks for hot start and cold accretion objects are shown in Fig. 8. The hot start tracks are taken from Burrows et al. (1997), while the cold start formation and evolution calculations for core accretion planets use the model of Mollière and Mordasini (2012). The objects shown in the figure have a mass ranging from 5 to $30 M_{\mathrm{J}}$. Note that the low-entropy tracks in Fig. 7 do not agree with those in Fig. 8: Fig. 7 assumes arbitrarily low post-formation entropies for cold start objects, whereas in Fig. 8 the low initial post formation entropies are determined self-consistently from the formation process. The point of Fig. 8 is that even objects formed by core accretion produce deuterium burning rates similar (but not identical) to those obtained by classical calculations which normally neglect the formation process, while the point of Fig. 7 is to show the differences in evolution between high and low entropy objects and how long it takes for both branches to converge. The formation is neither considered nor shown to focus on the effects of evolution.

The effect of the deuterium burning delaying the hot start's luminosity decrease for masses $>13 M_{\mathrm{J}}$ can clearly be seen in Fig. 8, as well as the increase in luminosity for cold start objects (in order to compare the hot and cold start calculations more easily, the cold start calculations were shifted in time by roughly $2 \mathrm{Myrs}$ ). For the cold start objects with masses $>20 M_{\mathrm{J}}$, the inflation of the planetary radius happens already during the formation phase of the planet, such that the increase in luminosity cannot be seen in the figure as it lies within the period where the powerful accretion shock luminosity dominates ( $<3$ Myrs in the figure). The accretion shock luminosity decreases a bit because of deuterium burning as the infalling gas is added at larger radii.

\subsection{Mass-Radius Relation}

A diagram that is of particular interest for planet formation and evolution theories is the planetary mass-radius diagram, as it contains information about the internal structure of (extrasolar) planets (e.g., Guillot 1999; Chabrier et al. 2009). It constrains the processes 
Fig. 9 Mass-radius diagram of observed and synthetic planets. The synthetic planets have an age of 5 Gyr and a semi-major axis $a$ between 0.1 and 1 AU. Observed planets with $a>0.1 \mathrm{AU}$ are included. The colors give the mass fraction of $\mathrm{H} / \mathrm{He}$ ranging from solid planets without $\mathrm{H} / \mathrm{He}$ (black) over ocher $(<1 \%)$ to dark orange $(>99 \%)$

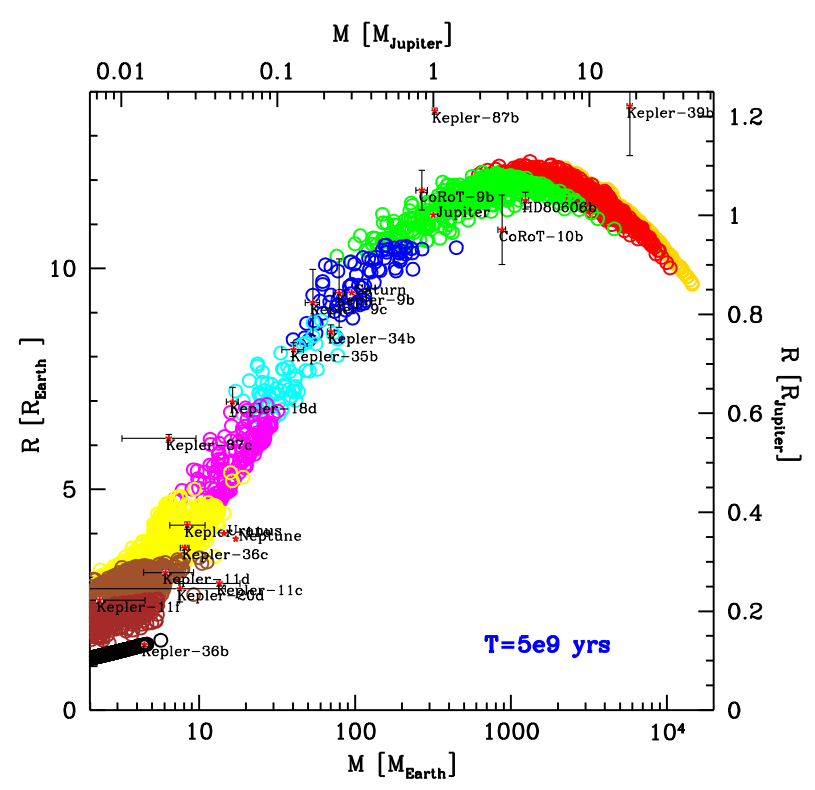

that determine the structure during the formation period (solid/gas accretion, orbital migration) as well as during the evolutionary phase (like atmospheric opacities, energy transport mechanisms, atmospheric escape, outgassing, or radius inflation).

Figure 9 compares the mass-radius relation obtained with a planet population synthesis calculation at an age of $5 \mathrm{Gyr}$ with observational data (updated from Mordasini et al. 2012b). The metallicity (core mass fraction) of the synthetic planets is color-coded in the figure. The chemical composition of the heavy elements depends on the planet's formation track. Heavy elements accreted beyond the iceline in the parent protoplanetary disk are assumed to consist of $50 \%$ water ice and $50 \%$ "rocky" material, while material accreted inside the iceline is fully "rocky", which is itself assumed to be made of $2 / 3$ silicates and $1 / 3$ iron. Such mass fractions are approximately expected for the condensation of solar composition gas (Lodders 2003; Santos et al. 2015). Although the observed exoplanets shown in Fig. 9 are not at an exact age of 5 Gyr the observational errors on the host star's age often allow for ages of about 5 Gyr. Furthermore, the planetary radius evolution in the Gyr time range is slow, such that the 5 Gyr snapshot should represent planets of various ages within the Gyr regime reasonably well.

The population synthesis model used in Fig. 9 self-consistently combines a planet formation model based on the core accretion paradigm (Alibert et al. 2005) with the long-term thermodynamical evolution of the planets after the dissipation of the protoplanetary disk (Mordasini et al. 2012a). The simulation includes the effect of atmospheric escape (see Sect. 4.5) but does not model radius inflation (see Sect. 4.6). To avoid planets which could be significantly inflated (Demory and Seager 2011), the minimum semi-major axis of the synthetic and observed planets included in the figure is $0.1 \mathrm{AU}$. mechanisms that could lead to inflated close-in exoplanets will be discussed in Sect. 4.6.

In general the synthetic mass-radius diagram has a specific shape that is characterized by an increase in radius with mass, which levels off and turns over at roughly $4 M_{\mathrm{J}}$. This maximum is due to an increase in the compressibility of the matter as the electron degeneracy increases. Furthermore, one sees that neither the upper-left corner in Fig. 9 (i.e., large lowmass planets) nor its lower-right corner (i.e., small massive planets) seem to be populated by 
Fig. 10 Mass fraction of $\mathrm{H} / \mathrm{He}$ relative to the total mass of a planet as a function of its total mass. The color-code gives the planetary radius in Earth radii. The larger the planet mass, the higher the mass fraction of the gas envelope. The change in slope at about $100 M_{\oplus}$ results from the transition between the Kelvin-Helmholtz contraction limited regime and the disk-limited gas accretion regime. This figure can be compared with observational constraints (see, e.g., Fig. 9 in Lopez and Fortney 2014)

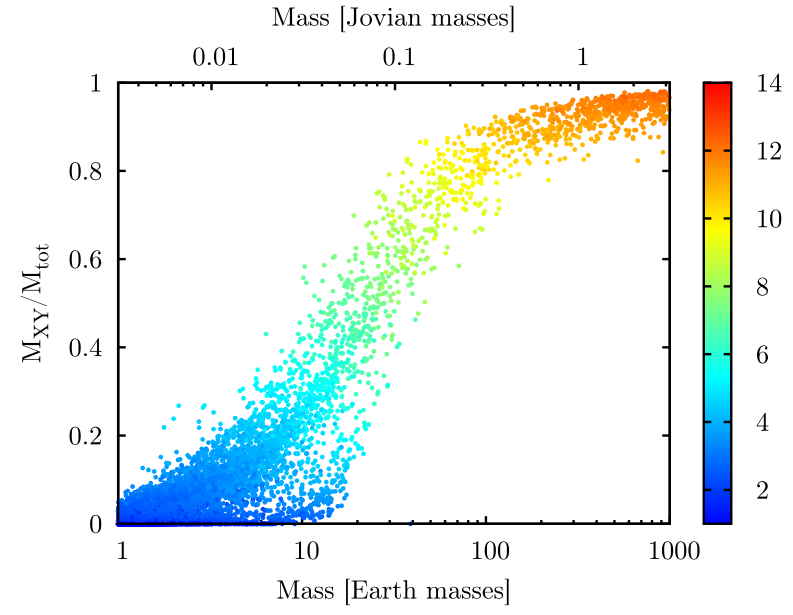

synthetic or observed planets. Synthetic planets with a low metal fraction have larger radii and masses than high-metallicity synthetic planets. At a given mass, the lower the radius, the more enriched is a synthetic planet, while planets of fixed enrichment have larger radii when their mass is higher, leading to diagonally colored bands in the synthetic mass-radius diagram.

The shape of the mass-radius diagram is a consequence of the formation process, in particular the resulting core and $\mathrm{H} / \mathrm{He}$ envelope masses, as well as material properties. The fraction of $\mathrm{H} / \mathrm{He}$ envelope mass as a function of the planet's total mass is displayed in Fig. 10. As the population synthesis is calculated under the core accretion paradigm, the planets start as low-mass solid cores, which can bind only tenuous gaseous envelopes due to long KH cooling timescales of their protoplanetary envelope during the nebular phase (Ikoma et al. 2000; Lee and Chiang 2015). Additionally, if the protoplanets are close to their star during formation, they will not accrete large amounts of gas due to the high local disk temperature (Ikoma and Hori 2012). Whatever gaseous envelope they accrete during formation can then be reduced again by envelope evaporation due to the stellar XUV insolation during evolution (e.g., Lammer et al. 2003; Owen and Jackson 2012; Lopez and Fortney 2013; Jin et al. 2014). Therefore, the lowest mass objects will have the highest heavy element content (essentially they are solid rocky or icy planets). As rocky or icy planets are only weakly compressible, their radius increases as their mass increases.

More massive planets can bind a larger gaseous envelope on top of their solid core. As they grow in mass, the amount of planetesimals which can be accreted increases as well due to the expansion of the feeding zone, but the net effect is still a decrease in the planetary metallicity (heavy element mass fraction) with mass $M$ roughly as $M^{-0.7}$ in the giant planet regime (Mordasini et al. 2014), in agreement with observational data (Miller and Fortney 2011).

Although the compressibility of gas is much higher than of solids, the planets still grow in radius for a given metallicity if the mass is increased. For ever higher solid core masses, the growing planets will eventually try to accrete and bind more gas from the protoplanetary disk than is locally available due to their very short KH timescales. This leads to a quasistatic collapse of their gaseous envelope and the so-called stage of runaway gas accretion (Bodenheimer et al. 2000). In this phase, the growing planets will accrete a large fraction of the gas delivered to their location by the viscous evolution of the protoplanetary disk (Lubow 
et al. 1999). The disk's lifetime and gas supply at the location of the planet then become the sole determining factors of the planets' final mass. The transition from the regime where the planet's KH-contraction controls gas accretion, to the one where the disk limits it, is visible in Fig. 10 by a change in slope at about $100 M_{\oplus}$. This figure can be compared to observational data where the $\mathrm{H} / \mathrm{He}$ mass fraction of actual exoplanets is derived from internal structure modeling (Fig. 9 in Lopez and Fortney 2014).

As it is inherently impossible to form low-mass gaseous planets without a core in the core accretion paradigm, no very large low-mass planets exist in the synthetic mass-radius diagram (nor actually in the observed diagram). Such planets would populate the upper-left corner in Fig. 9 (and Fig. 10). The presence of a massive core always leads to comparatively smaller radii for low-mass planets, even if there is a gaseous envelope on top of the core. On the other hand, massive solid planets will always undergo runaway gas accretion in the disk, such that it is impossible to form small (i.e., bare rocky or icy) but massive planets in the core accretion picture. This explains why the lower-right corner of Fig. 9 (and Fig. 10) also remains empty.

We see that the majority of the observed exoplanets fall within the mass-radius diagram obtained with the population synthesis. Kepler-87b and Kepler-39b are notable exceptions, being larger than the predicted synthetic radii of any given mass. The structure of these planets remains to be explained (Bouchy et al. 2011). The large radius of Kepler-39b could be caused by rapid rotation, its rotation period being estimated at only $1.6 \pm 0.4$ hours (Zhu et al. 2014). It is interesting to note that some objects similar to Kepler-39b in terms of mass, but at even higher insolation, follow the prediction of population syntheses much better (like CoRoT $3 b$, which has a mass of $21.7 \pm 1.0 M_{\mathrm{J}}$ and a radius of $1.01 \pm 0.07 R_{\mathrm{J}}$, see Moutou et al. 2013; Deleuil et al. 2008).

The fact that some of the observed exoplanets with a mass comparable to that of Neptune seem to be smaller than predicted by the model needs to be explained as well. There are two potential explanations. A first possibility is a higher opacity in the protoplanetary envelope during the formation phase. The accretion of $\mathrm{H} / \mathrm{He}$ during formation is regulated by the envelope's ability to cool, which is in turn mostly controlled by the opacity caused by small grains suspended in the planet's upper radiative zone. If the opacity is higher than assumed in this population synthesis (it is taken here equal to the ISM opacity multiplied by a factor of 0.003 , Mordasini et al. 2014), the planets would have smaller radii at a given mass due to a lower $\mathrm{H} / \mathrm{He}$ fraction. This shows how the planetary massradius relation can be used to constrain the microphysical models of the grain dynamics during formation like coagulation, settling and evaporation (Podolak 2003; Ormel 2014; Mordasini 2014). A second possibility is a more efficient envelope evaporation during evolution. If the loss of the primordial $\mathrm{H} / \mathrm{He}$ envelopes is actually more efficient than in the simple evaporation model taken here (e.g., because of different evaporation regimes applying to planets of different types, Owen and Alvarez 2016), this would also lead to smaller radius at a given mass. These two scenarios could potentially be disentangled by their different dependence on the planet's orbital distance, the planet's atmospheric composition, or on time. But in order to see such features, a high number of well-characterized planets is likely necessary, highlighting the importance of future missions like CHEOPS (Broeg et al. 2013) or PLATO (Rauer et al. 2014).

\subsection{The Formation of Close-in Low-Mass Planets, Envelope Evaporation, and the Planetary Mass-Density Diagram}

Close-in low-mass planets are extremely common around Sun-like stars with about 45-55\% of FGK stars having a planet with $M \lesssim 30 M_{\oplus}$ and a period $P<100$ days, a result from 
Table 1 Predictions of three scenarios for the formation of close-in low-mass planets in terms of the resulting core composition of the planets, whether planets attain a primordial $\mathrm{H} / \mathrm{He}$ envelope, are expected to be in mean motion resonances, and the disk mass necessary to form the planets. The term isolation refers to the situation where the planetesimals' orbits are sufficiently circularized by the protoplanetary gas disk so that they can never grow enough in mass by collisions and cannot attract a large envelope of gas during their formation

\begin{tabular}{|c|c|c|c|}
\hline \multirow[t]{2}{*}{ Quantity } & \multicolumn{3}{|l|}{ Scenario } \\
\hline & $\begin{array}{l}\text { Strictly in situ/ } \\
\text { no migration }\end{array}$ & $\begin{array}{l}\text { Weak migration, } \\
\text { then final assembly }\end{array}$ & $\begin{array}{l}\text { Assembly at } a \gtrsim a_{\text {ice }} \\
\text { large scale migration }\end{array}$ \\
\hline Core composition & Rocky & $\begin{array}{l}\text { Rocky to icy with } \\
\text { potential gradient }\end{array}$ & Icy \\
\hline Primordial H/He & $\begin{array}{l}\text { Yes (w/o isolation) } \\
\text { No (w isolation) }\end{array}$ & $\begin{array}{l}\text { Yes (w/o isolation) } \\
\text { No (w isolation) }\end{array}$ & Yes \\
\hline Mean-Motion Resonance & No & $\mathrm{No} / \mathrm{Yes}$ & Yes/No (see Sect. 3.3.2) \\
\hline Necessary disk mass & High & Intermediate & Low \\
\hline
\end{tabular}

high-precision RV surveys (Udry and Mayor 2008; Mayor et al. 2011) that was subsequently confirmed and extended by the Kepler survey (Borucki et al. 2011a).

Despite their high frequency, the formation mechanism of this class of planets (without analogue in the Solar system) is currently debated in the literature (e.g., Hansen and Murray 2012; Chiang and Laughlin 2013; Ida et al. 2013; Raymond and Cossou 2014; Schlichting 2014; Hands et al. 2014; Chatterjee and Tan 2014; Ogihara et al. 2015; Inamdar and Schlichting 2015 and Sect. 3.3.2). Some models propose an in situ formation at the current location of the planets close to the star, other models propose that the planets have originally formed further away, potentially in the colder regions of the protoplanetary disk beyond the water ice line, while some other models finally are of a hybrid type where at least some migration of the planets themselves or their building blocks has occurred. As summarized in Table 1, these models differ in their predictions not only regarding the architecture of the planetary systems (e.g., mean motion resonances, concentration towards the inner edge, relative size or mass of the planets), but also in their predicted bulk composition (ice mass fraction in the planetary core, $\mathrm{H} / \mathrm{He}$ mass fraction).

The table makes clear that knowing the bulk composition (H/He content; rocky, icy, mixed core composition) and also the atmospheric composition would be of very high interest to disentangle the different formation mechanisms. The different bulk compositions express themselves in different mean planetary densities, even if degeneracies unfortunately prevent a unique conversion of the observed mean density into an underlying bulk composition, at least for some parameters (e.g., Adams et al. 2008; Dorn et al. 2015).

Figure 11 shows the planetary mass-mean density diagram obtained in a population synthesis calculation based on the core accretion paradigm and assuming Sun-like stars (Swoboda et al., in prep.). During the formation phase, the concurrent formation of 10 embryos was calculated using the model of Alibert et al. (2013), while during the subsequent longterm evolution, the effects of cooling and contraction as well as atmospheric escape were included (Mordasini et al. 2012a; Jin et al. 2014). Atmospheric escape is an important process for low-mass close-in planets because (i) they are more susceptible to it than giant planets due to their weaker gravitational potential, and (ii) the loss of even a tenuous $\mathrm{H} / \mathrm{He}$ envelope strongly decreases their radius (e.g., Lopez and Fortney 2013). Envelope evaporation has been observed both for giant and low-mass planets (Vidal-Madjar et al. 2008; Ehrenreich et al. 2015). The mass-density diagram is interesting because it reveals more clearly than the mass-radius diagram the imprint of several prominent physical processes 


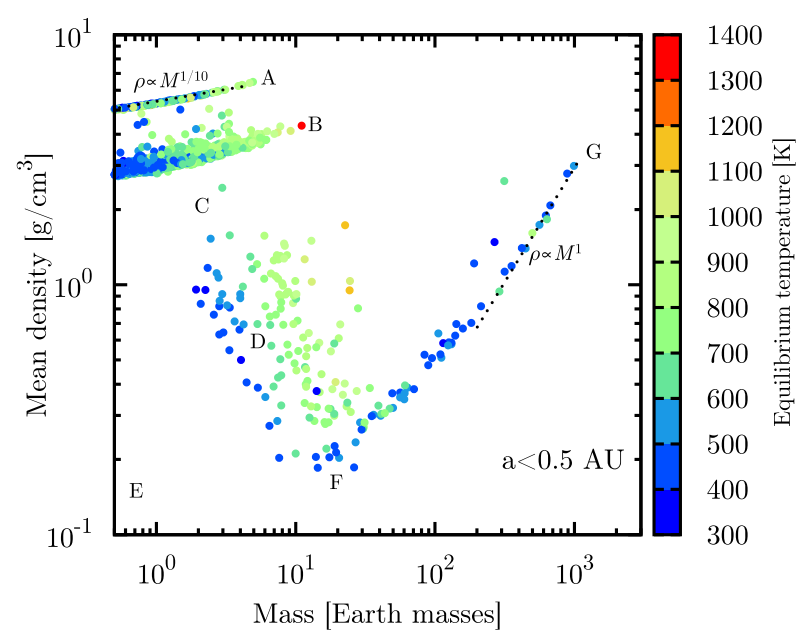

Fig. 11 Mean planetary density $\bar{\rho}$ as a function of mass $M$ in a synthetic population around Sun-like stars at an age of $5 \mathrm{Gyr}$. Planets have a semi-major axis $a<0.5 \mathrm{AU}$. The color bar gives the equilibrium temperature of the planets. Important features are: (A) bare rocky planets; (B) bare icy planets; (C) the evaporation valley; (D) low-mass core-dominated planets with $\mathrm{H} / \mathrm{He}$; (E) a forbidden zone because of evaporation $(a<0.5 \mathrm{AU})$; (F) the transition point to giant planets; (G) the giant planet branch. The analytic scaling $\bar{\rho} \propto M^{q}$ for a polytropic index of $n=1 / 7(q=1 / 10)$ applying to terrestrial planets and $n=1(q=1)$ for partially degenerate giant planets are overplotted by dotted lines

during planet formation and evolution (for a comparison with an observational $M-\bar{\rho}$ diagram, see Hatzes and Rauer 2015). They can be identified with seven features in Fig. 11:

A: bare rocky planets. These are low-mass rocky planets which have either started without an $\mathrm{H} / \mathrm{He}$ envelope (very low-mass planets) or which have lost it completely due to atmospheric escape. These planets have formed inside the iceline but have migrated to their current orbital distance. In the simulation, they fall on one single mass-density relation as a universal mass fraction of iron (1/3) was assumed in the synthesis, whereas in reality a certain spread is expected due to varying stellar compositions (Dorn et al. 2015) and to giant impacts which can partially remove the mantle (Benz et al. 2007). The colors show that at smaller orbital distances (corresponding to higher equilibrium temperatures $T_{\text {eq }}$ ), more massive planets have lost all their primordial $\mathrm{H} / \mathrm{He}$. This illustrates that the dividing line between solid and gaseous planets depends on orbital distance if it is only due to escape, which is however unlikely (Schlichting et al. 2015; Rogers 2015).

B: bare icy planets. These are low-mass planets formed outside the iceline which have migrated close to their host star and then have lost their $\mathrm{H} / \mathrm{He}$ due to evaporation. In the syntheses of Alibert et al. (2013), the planetary ice-mass fraction increases with increasing orbital distance and planetary mass, as more massive planets migrate faster in type I migration, and get more easily lost out of type I migration convergence zones ("traps") due to saturation of the corotation torque (e.g., Baruteau et al. 2014; Dittkrist et al. 2014 and Sect. 3.1.2). The mass-density relation is less narrow than for the bare rocky planets indicating a spread in the ice mass fraction, despite the fact that the increase in the planetesimals' surface density at the iceline (a factor of 2) was the same in all synthetic disks. This means that all planets accreting outside of the iceline start with an identical ice mass fraction in the core. The final spread is then a consequence of the accretion of rocky plan- 
etesimals during the migration through the inner disk (see Figueira et al. 2009 for the case of GJ436b).

C: evaporation valley. The evaporation valley separates planets without $\mathrm{H} / \mathrm{He}$ from lowmass planets that still have retained some H/He (Owen and Wu 2013; Lopez and Fortney 2013; Jin et al. 2014). It comes about as a small amount of $\mathrm{H} / \mathrm{He}$ (just $0.1-1 \%$ in mass) is sufficient to decrease $\bar{\rho}$ by a factor 2 or so, and because the timescale over which this last $\mathrm{H} / \mathrm{He}$ is lost is short $\left(\sim 10^{5}\right.$ years $)$ compared to the planet's lifetime. This means that it is not likely that at a given moment in time, like here 5 Gyrs, many planets are observed during the process of losing the last $\mathrm{H} / \mathrm{He}$, leading to the depletion of points in the valley.

D: low-mass core-dominated planets with $\mathbf{H} / \mathbf{H e}$. These are planets in the super-Earth mass range. They can have both icy or rocky cores depending on their formation history. A clear imprint of evaporation is seen: the hotter a planet, the larger its density at a given total mass as more primordial $\mathrm{H} / \mathrm{He}$ is lost for closer-in planets.

E: forbidden zone because of evaporation. This lower left triangle remains empty as only planets with $a<0.5 \mathrm{AU}$ are included, and at such distances, low-mass, very low-density planets would undergo intense evaporation. Therefore, these planets have already moved to higher mean densities by 5 Gyr. This means that the parameter space is in principle not only three-dimensional $\left(M, \bar{\rho}, T_{\text {eq }}\right)$, but that time should be an extra dimension.

F: transition point to giant planets. The point of lowest mean density in the diagram can be defined as the transition point from solid-dominated to gas-dominated planets. It occurs at a mass of $10-30 M_{\oplus}$, which corresponds to the mass where rapid gas accretion starts in the core-accretion model (Pollack et al. 1996). In the planetary mass function, a break in the slope can be seen at such a mass in both the theoretical and observational data (Mordasini et al. 2009b; Mayor et al. 2011).

G: giant planet branch. With further increasing mass, planets become denser again as the self-gravitational compaction becomes stronger for these gas-dominated, more compressible planets. In this regime, closer-in planets have a lower density (even if this is not clearly visible in this specific synthesis due to the low number of giant planets inside of $0.5 \mathrm{AU}$ ), which is the opposite to the core-dominated planets with $\mathrm{H} / \mathrm{He}$. For giant plants, the change of the atmospheric boundary conditions for more strongly irradiated planets delays somewhat the contraction (Guillot and Showman 2002), resulting in lower densities at higher $T_{\text {eq }}$. Additional radius inflation mechanisms (not included in this synthesis) tend to render this correlation even clearer (e.g., Laughlin et al. 2011).

Other features in the mass-mean density diagram, in particular the mass scalings, can be understood by considering polytropic models for planetary interiors for which the pressure $P$ depends on the density $\rho$ as $P \propto \rho^{1+1 / n}$, with $n$ the polytropic index (Chandrasekhar 1939; Chabrier et al. 2009). For such models, the mean density scales with mass as $\bar{\rho} \propto$ $M^{2 n /(3-n)}$.

In the giant planet regime the electrons are partially degenerate due to the relatively low temperatures and high densities. But the degeneracy is not complete so that both the quantum electron-degeneracy pressure and the classical electrostatic contribution from the non-degenerate ions matter. The polytropic index is therefore $n=1$ which corresponds to a (mean) density that increases linearly with mass $(\bar{\rho} \propto M)$ or, equivalently, to a radius that is independent of mass. Indeed, such a behavior is seen in the numerical simulations, as shown by the right dotted line close to Feature $\mathrm{G}$ (the giant planet branch). At lower masses, the degeneracy decreases and the composition changes from gas-dominated to solid-dominated planets. These planets are described by equations of state which are much less compressible.

For completely incompressible matter $(n=0)$, the mean density would be independent of mass, corresponding to a horizontal line in Fig. 11. Such a regime is approached in the case 
Table 2 Power-law indices governing the structure of various types of planets described by polytropic models (see text): $n=1 /(\gamma-1)$ is the polytropic index, $\gamma$ the adiabatic index, $p=(1-n) /(3-n)$ describes the power-law dependence between radius and mass, $q=2 n /(3-n)$ that between mean density and mass, and $\chi_{\text {norm }}=1 / \gamma$ that between mean density and pressure

\begin{tabular}{lllll}
\hline Planet type & Quantity & & \\
\cline { 2 - 5 } & $\begin{array}{l}\text { Polytropic } \\
\text { index } n\end{array}$ & $p=\partial \log R / \partial \log M$ & $q=\partial \log \bar{\rho} / \partial \log M$ & $\chi_{\text {norm }}=\partial \log \bar{\rho} / \partial \log P$ \\
\hline Incompressible & 0 & $1 / 3$ & 0 & 0 \\
Terrestrial planets & $1 / 7$ & $3 / 10$ & $1 / 10$ & $1 / 8$ \\
Giant planets & 1 & 0 & 1 & $1 / 2$ \\
Low mass BDs & $6 / 5$ & $-1 / 9$ & $4 / 3$ & $6 / 11$ \\
High mass BDs & $3 / 2$ & $-1 / 3$ & 2 & $3 / 5$ \\
\hline
\end{tabular}

of low-mass solid planets. The small, but still non-zero compressibility of rocks, ices, and iron however causes a weak increase of the density with mass also in this regime (see Features $\mathrm{A}$ and $\mathrm{B})$. For low-mass solid planets, the mass-radius relation scales approximately as $R \propto M^{3 / 10}$ (Grasset et al. 2009), corresponding to $n=1 / 7$, and therefore $\bar{\rho} \propto M^{1 / 10}$ which is a weak increase. Such scaling is shown by the left dotted line in Fig. 11. It approximately reproduces the results found by solving directly the interior structure equations of bare rocky or icy planets (Mordasini et al. 2012b).

The scaling relations for polytropic models are summarized in Table 2. The results are shown for planets that are approximately incompressible (very low-mass planets), terrestrial planets, partially degenerate giant planets with masses roughly between 1 and $10 M_{\mathrm{J}}$, more strongly degenerate high-mass giant planets and low-mass brown dwarfs with masses from about 10 to $30 M_{\mathrm{J}}$, and finally high-mass brown dwarfs which can be approximated with a fully degenerate non-relativistic equation of state $(n=3 / 2)$. The table gives the polytropic index $n$, the power-law exponent $p$ for the radius $R$ as function of mass $M\left(R \propto M^{p}\right)$, which, from the polytropic Lane-Emden equation, is linked to $n$ via $p=(1-n) /(3-n)$. The table also gives the power-law exponent $q$ for the mean density as function of mass $(\bar{\rho} \propto$ $\left.M^{q}\right)$, given by $q=2 n /(3-n)$, and finally the dimensionless power-law compressibility $\chi_{\text {norm }}=\partial \log \bar{\rho} / \partial \log P$, i.e. $\chi_{\text {norm }}=n /(n+1)=1 / \gamma(\gamma$ denotes the adiabatic index $)$. This is a good measure of the compressibility because it shows that for small values of $\chi_{\text {norm }}$ the density will only increase weakly even if the pressure increases strongly. For large values of $\chi_{\text {norm }}$, small changes in the pressure can change the density substantially. The quantity $\chi_{\text {norm }}$ increases with increasing mass. In reality, the transition from one regime to the other is smooth, as can be seen in Fig. 9. In this figure we see for example that, for low-mass giant planets, the radius increases with mass indicating an effective $n<1$, followed by the maximum at about $4 M_{\mathrm{J}}$ where $n=1$, and finally a smooth increase to even higher $n$ for more massive objects that only ends when hydrogen burning sets in at about 80 Jovian masses.

\subsection{Radius inflation of Hot Jupiters}

Radius inflation is a distinctive feature of close-in giant planets which was not expected theoretically and which is still not fully understood. With a radius of about $1.38 R_{\mathrm{J}}$, HD 209458 b, the first detected transiting extrasolar planet (Henry et al. 2000; Charbonneau et al. 2000), is significantly larger than expected from theoretical evolution models, even when the effects of stellar irradiation are taken into account (Guillot and Showman 2002). This can 
Fig. 12 Time evolution of the radius of a HD 209458 b-like planet. Results are shown for an isolated planet without stellar irradiation, an irradiated planet without inflation, and for four irradiated planet models where a fraction between 0.1 and $5 \%$ of the incoming stellar flux is added to the planet's luminosity in the deep convective zone. The red box indicates the observed radius and age of HD $209458 \mathrm{~b}$

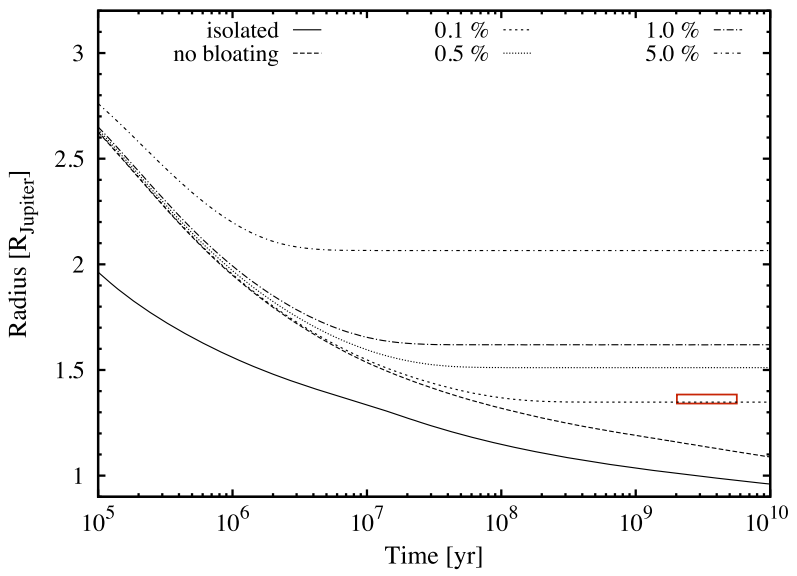

be seen in Fig. 9 which shows that standard cooling models do not predict the existence of planets with radii larger than about $1.1 R_{\mathrm{J}}$ (or up to $1.3 R_{\mathrm{J}}$ if smaller orbital distances are included) at ages of a few Gyrs. Yet, a significant fraction of hot Jupiters are larger than above threshold values, with some extreme cases such as HAT-P-32 b $\left(R=1.79 \pm 0.03 R_{\mathrm{J}}\right.$, Hartman et al. 2011) and WASP $17 \mathrm{~b}\left(R=1.93 \pm 0.08 R_{\mathrm{J}}\right.$, Anderson et al. 2010). The latter has a mean density about 10 times smaller than Jupiter's.

At the time of writing, thanks to a fairly large sample of transiting giant planets with a wide range of orbital distances, it is fairly well established that there exists a correlation between inflation and the intensity of stellar irradiation (Laughlin et al. 2011). It is found that modestly irradiated planets do not have inflated radii and that below a threshold value for the incident flux of about $2 \times 10^{8} \mathrm{erg} \mathrm{s}^{-1} \mathrm{~cm}^{-2}$ (corresponding to a solar-like star at an orbital distance of about $0.08 \mathrm{AU}$ ) radius inflation ceases (Demory and Seager 2011).

The observation that radius inflation, also called radius anomaly, is correlated with the incident stellar flux may therefore indicate that some of the incident stellar energy is deposited deep inside the planets' interior, thereby delaying or even halting their contraction. For this to work, part of the stellar irradiation flux must be converted into some other form of energy that is then deposited in the deeper layers, as pure irradiation of the planet's atmosphere by the star does not penetrate deep enough into the planet to cause a large enough inflation (Burrows et al. 2000) - it is instead radiated back to space. From evolutionary models, one finds that $0.1-1 \%$ of the stellar flux impinging on the planet's atmosphere is sufficient to explain the radius anomaly at the current age of the planets if it can penetrate to the deep convective zone of the planets (Guillot and Showman 2002).

This is illustrated in Fig. 12, which displays the time evolution of the radius of a planet with parameters similar to HD $209458 \mathrm{~b}\left(M=0.69 \pm 0.02 M_{\mathrm{J}}, a=0.0475 \pm 0.0006 \mathrm{AU}\right.$, age $=4.0 \pm 2.0 \mathrm{Gyr})$. The results of six models are illustrated in the figure: an isolated planet (without stellar irradiation), an irradiated planet for which stellar irradiation does not come as an additional energy source for the planet (no bloating, that is no radius inflation), and for four irradiated planets for which a fraction of the incoming stellar flux is added to the planet's intrinsic luminosity at the center (thereby inflating the planet). According to these calculations, which use the planet evolution model of Mordasini et al. (2015), stellar irradiation increases the radius relative to the isolated case at late times by about $0.13 R_{\mathrm{J}}$, bringing it to $1.13 R_{\mathrm{J}}$ at $4 \mathrm{Gyr}$, which is clearly less than the observed radius (marked by the red box in Fig. 12). These calculations indicate that about $0.1 \%$ of the incoming flux 
must be added to the planet luminosity in the deep convective zone in order to reproduce approximately the observed radius (for comparison, Guillot and Showman 2002 found about $0.08 \%)$.

We note that a difference in radius of about $0.25 R_{\mathrm{J}}$ between the standard irradiated model and the aforementioned inflated model corresponds to a much hotter thermodynamics state of the planet as the partially degenerate equation of state governing the planet's interior means that the radius is only weakly dependent on temperature. This is visible from the result that the irradiated planet without additional energy sources contracted to the observed radius when the planet was almost 100 times younger than the actual age of the system. The $0.1 \%$ fraction of the incoming stellar irradiation necessary to bring the planet to its observed radius corresponds to a luminosity that is about 90 times bigger than the presentday luminosity of a planet undergoing standard cooling without inflation.

There are two main mechanisms that can turn stellar irradiation into an energy flux reaching the deep interior of planets, but note that none of them can so far explain the radius anomaly of all extrasolar planets quantitatively (Baraffe et al. 2014):

- The first one is the so-called ohmic heating mechanism, for which ionized alkali atoms in the winds caused by stellar irradiation of the planet produce an electric current that is dissipated in the deep layers of the planet (Batygin and Stevenson 2010; Perna et al. 2010). However, the study by Huang and Cumming (2012), who calculated the heating rate more self-consistently than earlier works, indicates that ohmic dissipation might not be strong enough to explain the radius anomaly of the very massive and very hot Jupiters.

- The second mechanism is the so-called weather noise mechanism (Showman and Guillot 2002). The strong winds resulting from the large temperature contrasts between the day- and night-sides of tidally locked planets are found in the numerical simulations of atmospheric circulation of Showman and Guillot (2002) to lead to a significant downward transport of kinetic energy. This energy is then dissipated in the deep layers, the details of which are currently not well understood. Also, the feasibility of a sufficiently large downward transport of kinetic energy depends on the details of the employed atmospheric circulation model and is subject to significant uncertainties (Baraffe et al. 2014). Lastly, the combination of tides together with the temperature contrast between the planetary day- and night-sides may produce work in the planet which could again result in an energy flux to the planet's deep layers (thermal tides, see Arras and Socrates 2010).

Other mechanisms have been proposed to explain the observed radius anomaly of hot Jupiters, which we briefly outline below:

- An increased metallicity in the planet's atmosphere which could lead to a slower contraction, because the opacity is expected to increase along with the metallicity of the planet and therefore to slow down its cooling (Burrows et al. 2007),

- A decrease in the efficiency of convective energy transport in the deep interior of the planet due to compositional gradients and the associated onset of semi-convection, which would also slow down the planets' cooling (Chabrier and Baraffe 2007),

- Tidal dissipation in the planets' interior resulting from star-planet tidal interactions (e.g., Bodenheimer et al. 2001). While tidal circularization of a giant planet (whose eccentricity could arise from interactions with unseen planetary companions) could provide significant heating in the early evolution of the planet, it is unlikely to remain a significant source of radius inflation at the typical ages where inflated hot Jupiters are observed (Leconte et al. 2010). Also, tidal dissipation does not account for the observed dependency between radius anomaly and stellar irradiation flux. 
In conclusion of this paragraph, the correlation between the occurrence of inflated hot Jupiters and stellar irradiation is well established observationally, but the mechanisms behind it remain poorly understood. Theorists have yet to come up with a convincing theory which can explain the observations and quantitatively work when coupled to planetary structure models.

\section{Summary}

The main points to take away from this chapter are the following:

- The planetesimal accretion scenario of planetary growth is dictated by self-gravity, and proceeds through runaway growth and oligarchic growth. It can account for terrestrial planet formation in the inner Solar system. Growth becomes inefficient in the oligarchic phase, and growth timescales become exceedingly long toward large orbital separations.

- Accretion of small pebbles is assisted by gas drag, and is most efficient for marginally coupled particles with stopping time to orbital period ratios between roughly 0.1 and 1 . Once a substantial fraction of solid mass resides in such pebbles, embryos that have grown across the transition mass accrete most pebbles entering their Hill sphere, and growth timescales are well within the lifetime of protoplanetary disks even at large orbital separations.

- Both planetesimal and pebble accretions likely play important roles in the formation of the Solar system as well as for exoplanets, and they may dominate at different phases and in different regions of protoplanetary disks. Global models of planet formation have begun to take both accretion scenarios into account. Pinning down the uncertainties requires better understandings of disk physics.

- The gravitational interaction between planets and their protoplanetary disk plays a prominent role in the early orbital evolution of planetary systems. Planet-disk interactions usually damp the eccentricity and inclination, but can lead to inward or outward migration depending on the planet's mass and, crucially, the disk's physical properties near the planet (radial profiles of the disk's density and temperature, magnetic field, turbulence). While tremendous progress has been made in the past few years in understanding the physics of planetary migration, especially that of low-mass planets, predictive scenarios of planet migration require more knowledge of protoplanetary disks in regions of planet formation. Pursuing detailed observations of protoplanetary disks as well as comprehensive models of the dynamics of such disks will help theories of disk migration become more predictive.

- While disk migration is certainly inevitable, it is one among many mechanisms that shape the architecture of planetary systems. The great diversity of extrasolar planetary systems shows that disk and high-eccentricity migrations, interactions with host and nearby stars all play some role. (Mis-)aligned hot Jupiters and the many multiple super-Earths systems illustrate the plurality of mechanisms shaping planetary systems.

- Evolutionary models establish the link between formation and observations. They are keys to estimate the mass of young directly imaged planets for which only the age and luminosity are known, and to learn about the interior composition and structure of planets when only their mass and radius are known like for close-in low-mass planets. For this class of planets, the bulk composition like the $\mathrm{H} / \mathrm{He}$ mass fraction and the ice mass fraction in the core represent important constraints for their formation mode(s). Atmospheric compositional measurements (using transit spectra) could help break degeneracies between composition and structure in the future, and help constrain the planet's formation mechanism and migration history. 
- The transition between giant planets and brown dwarfs is smooth. Planets with a mass of $\sim 13 M_{\mathrm{J}}$ can burn deuterium in their interior, just like brown dwarfs. After a rapid phase of deuterium burning, a non deuterium-burning planet of $12 M_{\mathrm{J}}$ and a planet that has depleted its deuterium (say, with a mass of $14 M_{\mathrm{J}}$ ) will have very similar structures, comparable to that of low-mass brown dwarfs after their deuterium is depleted (except, for a solid core which can, however, dissolve into the $\mathrm{H} / \mathrm{He}$ in time).

- Like for models of planet migration, predictions of evolutionary models are tied to the formation process (e.g., hot or cold start models), especially for young planets. While many exoplanets are well described by the mass-radius or the mass-mean density distributions predicted by core accretion linked to evolutionary models, there are several ways such models can be improved, in particular regarding the planet's internal structure (e.g., the occurrence of semi-convection), its material properties (via updated equations of states at high pressures and temperatures), and models for special evolutionary effects like atmospheric escape or radius inflation.

Acknowledgements We thank the organizers of the ISSI workshop "The Disk in Relation to the Formation of Planets and their Protoatmospheres", which was held in Beijing in August 2014. We thank D. Lin, N. Madhusudhan, Z. Sandor and S. Udry for stimulating discussions at the workshop. We thank Gabriel Marleau for interesting discussions and for providing Figure 7, and David Swoboda and Yann Alibert for providing the starting data for Figure 11. We also thank Aurélien Crida and Bertram Bitsch for detailed comments on an earlier draft of this paper, and the referee for a detailed and constructive report. XNB acknowledges support from Hubble and ITC Fellowships. CM acknowledges the support from the Swiss National Science Foundation under grant BSSGI0_155816 "PlanetsInTime".

\section{References}

E.R. Adams et al., Astrophys. J. 673, 1160 (2008)

S. Albrecht et al., Astrophys. J. 757, 18 (2012)

Y. Alibert et al., Astron. Astrophys. 434, 343 (2005)

Y. Alibert et al., Astron. Astrophys. 558, A109 (2013)

ALMA Partnership et al., Astrophys. J. Lett. 808, L3 (2015)

D.R. Anderson et al., Astrophys. J. 709, 159 (2010)

P.J. Armitage, Astrophysics of Planet Formation (2010)

P. Arras, A. Socrates, Astrophys. J. 714, 1 (2010)

X.-N. Bai, Astrophys. J. 798, 84 (2015)

X.-N. Bai, J.M. Stone, Astrophys. J. 769, 76 (2013)

X.-N. Bai, J.M. Stone, Astrophys. J. 796, 31 (2014)

I. Baraffe et al., Astron. Astrophys. 402, 701 (2003)

I. Baraffe et al., Astron. Astrophys. 482, 315 (2008)

I. Baraffe et al., in Protostars and Planets VI (2014), pp. 763-786

A.J. Barker, Y. Lithwick, Mon. Not. R. Astron. Soc. 437, 305 (2014)

C. Baruteau, F. Masset, Astrophys. J. 672, 1054 (2008)

C. Baruteau, F. Masset, in Lecture Notes in Physics, vol. 861, ed. by J. Souchay, S. Mathis, T. Tokieda (Springer, Berlin, 2013), p. 201

C. Baruteau, J.C.B. Papaloizou, Astrophys. J. 778, 7 (2013)

C. Baruteau et al., Astron. Astrophys. 533, A84 (2011a)

C. Baruteau et al., Mon. Not. R. Astron. Soc. 416, 1971 (2011b)

C. Baruteau et al., in Protostars and Planets VI (2014), pp. 667-689

M.R. Bate et al., Mon. Not. R. Astron. Soc. 401, 1505 (2010)

K. Batygin, Nature 491, 418 (2012)

K. Batygin, D.J. Stevenson, Astrophys. J. Lett. 714, L238 (2010)

K.R. Bell, D.N.C. Lin, Astrophys. J. 427, 987 (1994)

M. Benisty et al., Astron. Astrophys. 578, L6 (2015)

P. Benítez-Llambay et al., Nature 520, 63 (2015)

O. Benomar et al., Publ. Astron. Soc. Jpn. 66, 94 (2014) 
W. Benz et al., Space Sci. Rev. 132, 189 (2007)

W. Benz et al., in Protostars and Planets VI (2014), pp. 691-713

B. Bitsch et al., Astron. Astrophys. 555, A124 (2013a)

B. Bitsch et al., Astron. Astrophys. 549, A124 (2013b)

B. Bitsch et al., Astron. Astrophys. 570, A75 (2014)

B. Bitsch et al., Astron. Astrophys. 582, A112 (2015a)

B. Bitsch et al., Astron. Astrophys. 575, A28 (2015b)

P.H. Bodenheimer, J.B. Pollack, Icarus 67, 391 (1986)

P.H. Bodenheimer et al., Icarus 143, 2 (2000)

P. Bodenheimer et al., Astrophys. J. Lett. 548, 466 (2001)

P. Bodenheimer et al., Astrophys. J. 770, 120 (2013)

A.C. Boley, Astrophys. J. Lett. 695, L53 (2009)

A.C. Boley et al., Icarus 207, 509 (2010)

W.J. Borucki et al., Astrophys. J. 728, 117 (2011a)

W.J. Borucki et al., Astrophys. J. 736, 19 (2011b)

A.P. Boss, Science 276, 1836 (1997)

A.P. Boss, Astrophys. J. 661, L73 (2007)

F. Bouchy et al., Astron. Astrophys. 533, A83 (2011)

C. Broeg et al., in European Physical Journal Web of Conferences, vol. 47 (2013), p. 3005

A. Burrows, J. Liebert, Rev. Mod. Phys. 65, 301 (1993)

A. Burrows et al., Astrophys. J. 491, 856 (1997)

A. Burrows et al., Astrophys. J. Lett. 534, L97 (2000)

A. Burrows et al., Rev. Mod. Phys. 73, 719 (2001)

A. Burrows et al., Astrophys. J. 661, 502 (2007)

D. Cébron et al., Icarus 226, 1642 (2013)

G. Chabrier, I. Baraffe, Annu. Rev. Astron. Astrophys. 38, 337 (2000)

G. Chabrier, I. Baraffe, Astrophys. J. Lett. 661, L81 (2007)

G. Chabrier et al., Am. Inst. Phys. Conf. Ser. 1094, 102 (2009)

G. Chabrier et al., in Protostars and Planets VI (2014), pp. 619-642

J.E. Chambers, Icarus 233, 83 (2014)

S. Chandrasekhar, An Introduction to the Study of Stellar Structure (The University of Chicago Press, Chicago, 1939)

W.J. Chaplin et al., Astrophys. J. 766, 101 (2013)

D. Charbonneau et al., Astrophys. J. Lett. 529, L45 (2000)

S. Chatterjee, J.C. Tan, Astrophys. J. 780, 53 (2014)

S. Chatterjee et al., Astrophys. J. 686, 580 (2008)

E. Chiang, G. Laughlin, Mon. Not. R. Astron. Soc. 431, 3444 (2013)

B. Commerçon et al., Astron. Astrophys. 530, 13 (2011)

A. Crida, K. Batygin, Astron. Astrophys. 567, A42 (2014)

A. Crida, A. Morbidelli, Mon. Not. R. Astron. Soc. 377, 1324 (2007)

A. Crida et al., Icarus 181, 587 (2006)

A. Crida et al., Astron. Astrophys. 502, 679 (2009)

T. Currie et al., Astrophys. J. Lett. 796, L30 (2014)

G. D’Angelo, S.H. Lubow, Astrophys. J. 685, 560 (2008)

G. D'Angelo et al., Mon. Not. R. Astron. Soc. 358, 316 (2005)

G. D'Angelo et al., Giant planet formation, in Exoplanets, ed. by S. Seager (University of Arizona Press, Tucson, 2011), pp. 319-346

M. Deleuil et al., Astron. Astrophys. 491, 889 (2008)

B.-O. Demory, S. Seager, Astrophys. J. Suppl. Ser. 197, 12 (2011)

H.E. Dewitt et al., Astrophys. J. 181, 439 (1973)

K.-M. Dittkrist et al., Astron. Astrophys. 567, A121 (2014)

R. Dong et al., Astrophys. J. 741, 57 (2011)

R. Dong et al., Astrophys. J. Lett. 809, L5 (2015)

C. Dorn et al., Astron. Astrophys. 577, A83 (2015)

C.D. Dressing et al., Astrophys. J. 800, 135 (2015)

P.C. Duffell, Astrophys. J. Lett. 807, L11 (2015)

P.C. Duffell, A.I. MacFadyen, Astrophys. J. 769, 41 (2013)

R.H. Durisen et al., in Protostars and Planets V (2007), pp. 607-622

C. Dürmann, W. Kley, Astron. Astrophys. 574, A52 (2015)

D. Ehrenreich et al., Nature 522, 459 (2015)

J.A. Eisner, Astrophys. J. Lett. 803, L4 (2015) 
C. Espaillat et al., in Protostars and Planets VI (2014), pp. 497-520

D. Fabrycky, S. Tremaine, Astrophys. J. 669, 1298 (2007)

S.M. Fendyke, R.P. Nelson, Mon. Not. R. Astron. Soc. 437, 96 (2014)

P. Figueira et al., Astron. Astrophys. 493, 671 (2009)

D.A. Fischer, J. Valenti, Astrophys. J. 622, 1102 (2005)

D. Forgan, K. Rice, Mon. Not. R. Astron. Soc. 432, 3168 (2013)

J.J. Fortney, N. Nettelmann, Space Sci. Rev. 152, 423 (2010)

J.J. Fortney et al., Astron. Nachr. 326, 925 (2005)

W.A. Fowler et al., Annu. Rev. Astron. Astrophys. 5, 525 (1967)

R.S. Freedman et al., Astrophys. J. Suppl. Ser. 174, 504 (2008)

S. Fromang et al., Mon. Not. R. Astron. Soc. 363, 943 (2005)

J. Fung et al., Astrophys. J. 782, 88 (2014)

M. Galvagni et al., Mon. Not. R. Astron. Soc. 427, 1725 (2012)

C.F. Gammie, Astrophys. J. 553, 174 (2001)

P. Goldreich et al., Annu. Rev. Astron. Astrophys. 42, 549 (2004)

H.C. Graboske et al., Astrophys. J. 181, 457 (1973)

H.C. Graboske et al., Astrophys. J. 199, 265 (1975)

O. Grasset et al., Astrophys. J. 693, 722 (2009)

R. Greenberg et al., Icarus 35, 1 (1978)

O. Gressel et al., Astrophys. J. 779, 59 (2013)

O. Gressel et al., Astrophys. J. 801, 84 (2015)

J. Guilet et al., Mon. Not. R. Astron. Soc. 430, 1764 (2013)

T. Guillot, Science 286, 72 (1999)

T. Guillot, Annu. Rev. Astron. Astrophys. 33(1), 493 (2005)

T. Guillot, D. Gautier, ArXiv e-prints (2014)

T. Guillot, A.P. Showman, Astron. Astrophys. 385, 156 (2002)

T. Guillot et al., Astrophys. J. 450, 463 (1995)

T. Guillot et al., The Interior of Jupiter (Cambridge University Press, Cambridge, 2004), pp. 35-57

K.E. Haisch Jr. et al., Astrophys. J. Lett. 553, L153 (2001)

T.O. Hands et al., Mon. Not. R. Astron. Soc. 445, 749 (2014)

B.M.S. Hansen, N. Murray, Astrophys. J. 751, 158 (2012)

B.M.S. Hansen, N. Murray, Astrophys. J. 775, 53 (2013)

L. Hartmann et al., Astrophys. J. 475, 770 (1997)

J.D. Hartman et al., Astrophys. J. 742, 59 (2011)

A.P. Hatzes, H. Rauer, Astrophys. J. Lett. 810, L25 (2015)

C. Hayashi, T. Nakano, Prog. Theor. Phys. 30, 460 (1963)

R. Helled et al., in Protostars and Planets VI (2014), pp. 643-665

G.W. Henry et al., Astrophys. J. Lett. 529, L41 (2000)

A.M. Hofmeister, R.E. Criss, Tectonophysics 395, 159 (2005)

X. Huang, A. Cumming, Astrophys. J. 757, 47 (2012)

W.B. Hubbard, Rev. Geophys. 18(1), 1 (1980)

D. Huber et al., Science 342, 331 (2013)

E. Iaroslavitz, M. Podolak, Icarus 187, 600 (2007)

S. Ida, D.N.C. Lin, Astrophys. J. 673, 487 (2008)

S. Ida, J. Makino, Icarus 106, 210 (1993)

S. Ida et al., Astrophys. J. 775, 42 (2013)

M. Ikoma, Y. Hori, Astrophys. J. 753, 66 (2012)

M. Ikoma et al., Astrophys. J. 537, 1013 (2000)

N.K. Inamdar, H.E. Schlichting, Mon. Not. R. Astron. Soc. 448, 1751 (2015)

S. Jin et al., Astrophys. J. 795, 65 (2014)

A. Johansen, P. Lacerda, Mon. Not. R. Astron. Soc. 404, 475 (2010)

J.A. Johnson et al., Publ. Astron. Soc. Pac. 122, 905 (2010)

A. Juhász et al., Mon. Not. R. Astron. Soc. 451, 1147 (2015)

M. Jurić, S. Tremaine, Astrophys. J. 686, 603 (2008)

Kamland Collaboration et al., Nat. Geosci. 4, 647 (2011)

G.M. Kennedy, S.J. Kenyon, Astrophys. J. 673, 502 (2008)

S.J. Kenyon, B.C. Bromley, Astron. J. 131, 1837 (2006)

S.J. Kenyon, B.C. Bromley, Astrophys. J. Lett. 690, L140 (2009)

R. Kippenhahn, A. Weigert, Stellar Structure and Evolution (1990)

W. Kley, A. Crida, Astron. Astrophys. 487, L9 (2008)

W. Kley, R.P. Nelson, Annu. Rev. Astron. Astrophys. 50, 211 (2012) 
W. Kley et al., Astron. Astrophys. 506, 971 (2009)

E. Kokubo, S. Ida, Icarus 114, 247 (1995)

E. Kokubo, S. Ida, Icarus 123, 180 (1996)

E. Kokubo, S. Ida, Icarus 131, 171 (1998)

E. Kokubo, S. Ida, Icarus 143, 15 (2000)

D.G. Korycansky, J.B. Pollack, Icarus 102, 150 (1993)

K.M. Kratter, G. Lodato, ArXiv e-prints (2016)

K.A. Kretke, H.F. Levison, Astron. J. 148, 109 (2014)

S.S. Kumar, Astrophys. J. 137, 1121 (1963)

A.-M. Lagrange et al., Astron. Astrophys. 493, L21 (2009)

A.-M. Lagrange et al., Science 329, 57 (2010)

D. Lai, Mon. Not. R. Astron. Soc. 423, 486 (2012)

M. Lambrechts, A. Johansen, Astron. Astrophys. 544, A32 (2012)

M. Lambrechts, A. Johansen, Astron. Astrophys. 572, A107 (2014)

H. Lammer et al., Astrophys. J. Lett. 598, L121 (2003)

G. Laughlin et al., Astrophys. J. Lett. 729, L7 (2011)

J. Leconte, G. Chabrier, Astron. Astrophys. 540, A20 (2012)

J. Leconte, G. Chabrier, Nat. Geosci. 6, 347 (2013)

J. Leconte et al., Astron. Astrophys. 516, A64 (2010)

E.J. Lee, E. Chiang, Astrophys. J. 811, 41 (2015)

E. Lega et al., Mon. Not. R. Astron. Soc. 440, 683 (2014)

G. Lesur et al., Astron. Astrophys. 566, A56 (2014)

D.N.C. Lin, J. Papaloizou, Astrophys. J. 307, 395 (1986)

Y. Lithwick, Y. Wu, Astrophys. J. Lett. 756, L11 (2012)

K. Lodders, Astrophys. J. 591, 1220 (2003)

E.D. Lopez, J.J. Fortney, Astrophys. J. 776, 2 (2013)

E.D. Lopez, J.J. Fortney, Astrophys. J. 792, 1 (2014)

S.H. Lubow et al., Astrophys. J. 526, 1001 (1999)

N. Madhusudhan et al., Astrophys. J. Lett. 794, L12 (2014)

M. Malik et al., Astrophys. J. 802, 56 (2015)

G.W. Marcy et al., Astrophys. J. Suppl. Ser. 210, 20 (2014)

G.-D. Marleau, A. Cumming, Mon. Not. R. Astron. Soc. 437, 1378 (2014)

M.S. Marley et al., Astrophys. J. 655, 541 (2007)

C. Marois et al., Nature 468, 1080 (2010)

F. Marzari et al., Astron. Astrophys. 514, L4 (2010)

F.S. Masset, Celest. Mech. Dyn. Astron. 111, 131 (2011)

F.S. Masset, P. Benítez-Llambay, Astrophys. J. 817, 19 (2016)

F.S. Masset, J. Casoli, Astrophys. J. 723, 1393 (2010)

F.S. Masset, J.C.B. Papaloizou, Astrophys. J. 588, 494 (2003)

M. Mayor et al., arXiv, astro-ph.EP (2011)

T. Mazeh et al., Astrophys. J. 801, 3 (2015)

N.K. Miller, J.J. Fortney, Astrophys. J. Lett. 736, L29 (2011)

H. Mizuno, Prog. Theor. Phys. 64, 544 (1980)

P. Mollière, C. Mordasini, Astron. Astrophys. 547, A105 (2012)

M. Montesinos et al., Astrophys. J. 806, 253 (2015)

A. Morbidelli, D. Nesvorny, Astron. Astrophys. 546, A18 (2012)

A. Morbidelli et al., Icarus 258, 418 (2015)

C. Mordasini, Astron. Astrophys. 558, A113 (2013)

C. Mordasini, Astron. Astrophys. 572, A118 (2014)

C. Mordasini et al., in Tenth Anniversary of 51 Peg-b: Status of Prospects for Hot Jupiter Studies, ed. by L. Arnold, F. Bouchy, C. Moutou (2006) pp. 84-86

C. Mordasini et al., Astron. Astrophys. 501, 1139 (2009a)

C. Mordasini et al., Astron. Astrophys. 501, 1161 (2009b)

C. Mordasini et al., Astron. Astrophys. 547, 111 (2012a)

C. Mordasini et al., Astron. Astrophys. 547, 112 (2012b)

C. Mordasini et al., Astron. Astrophys. 566, A141 (2014)

C. Mordasini et al., Int. J. Astrobiol. 14, 201 (2015)

C. Moutou et al., Icarus 226, 1625 (2013)

T. Muto et al., Astrophys. J. 724, 448 (2010)

S. Naoz et al., Astrophys. J. Lett. 754, L36 (2012)

A. Natta et al., in Protostars and Planets V, ed. by B. Reipurth, D. Jewitt, K. Keil (2007), pp. 767-781 
S. Nayakshin, Mon. Not. R. Astron. Soc. 408, L36 (2010)

S. Nayakshin, S.-H. Cha, Mon. Not. R. Astron. Soc. 435, 2099 (2013)

R. Neuhäuser, T.O.B. Schmidt, ArXiv e-prints (2012)

M. Ogihara et al., Astron. Astrophys. 578, A36 (2015)

G.I. Ogilvie, Annu. Rev. Astron. Astrophys. 52, 171 (2014)

C.W. Ormel, Astrophys. J. Lett. 789, L18 (2014)

C.W. Ormel, H.H. Klahr, Astron. Astrophys. 520, A43 (2010)

J.E. Owen, M.A. Alvarez, Astrophys. J. 816, 34 (2016)

J.E. Owen, A.P. Jackson, Mon. Not. R. Astron. Soc. 425, 2931 (2012)

J.E. Owen, Y. Wu, Astrophys. J. 775, 105 (2013)

S.-J. Paardekooper, G. Mellema, Astron. Astrophys. 459, L17 (2006)

S.-J. Paardekooper, J.C.B. Papaloizou, Astron. Astrophys. 485, 877 (2008)

S. Paardekooper et al., Mon. Not. R. Astron. Soc. 401, 1950 (2010)

S. Paardekooper et al., Mon. Not. R. Astron. Soc. 410, 293 (2011)

S.-J. Paardekooper et al., Mon. Not. R. Astron. Soc. 434, 3018 (2013)

S. Paardekooper, Mon. Not. R. Astron. Soc. 444, 2031 (2014)

J.C.B. Papaloizou, Celest. Mech. Dyn. Astron. 111, 83 (2011)

J.C.B. Papaloizou et al., Mon. Not. R. Astron. Soc. 350, 829 (2004)

A. Pepliński et al., Mon. Not. R. Astron. Soc. 386, 164 (2008)

H.B. Perets, R.A. Murray-Clay, Astrophys. J. 733, 56 (2011)

S. Perez et al., Astrophys. J. Lett. 811, L5 (2015)

R. Perna et al., Astrophys. J. 724, 313 (2010)

F. Perri, A.G.W. Cameron, Icarus 22, 416 (1974)

G. Picogna, F. Marzari, Astron. Astrophys. 583, A133 (2015)

A. Pierens, Mon. Not. R. Astron. Soc. 454, 2003 (2015)

A. Pierens et al., Astron. Astrophys. 531, A5 (2011)

M. Podolak, Icarus 165, 428 (2003)

J.B. Pollack et al., Icarus 30, 111 (1977)

J.B. Pollack et al., Icarus 124, 62 (1996)

T. Prodanović et al., Mon. Not. R. Astron. Soc. 406, 1108 (2010)

S.P. Quanz et al., Astrophys. J. 807, 64 (2015)

R.R. Rafikov, Astrophys. J. 572, 566 (2002)

R.R. Rafikov, Astron. J. 128, 1348 (2004)

R.R. Rafikov, Astrophys. J. Lett. 621, L69 (2005)

F.A. Rasio, E.B. Ford, Science 274, 954 (1996)

H. Rauer et al., Exp. Astron. 38, 249 (2014)

S.N. Raymond, C. Cossou, Mon. Not. R. Astron. Soc. 440, L11 (2014)

S.N. Raymond et al., in Protostars and Planets VI (2014), pp. 595-618

H. Rein, Mon. Not. R. Astron. Soc. 427, L21 (2012)

L. Ricci et al., Astron. Astrophys. 512, A15 (2010)

J. Rodmann et al., Astron. Astrophys. 446, 211 (2006)

L.A. Rogers, Astrophys. J. 801, 41 (2015)

T.M. Rogers, D.N.C. Lin, Astrophys. J. Lett. 769, L10 (2013)

R. Sanchis-Ojeda et al., Astrophys. J. 733, 127 (2011)

N.C. Santos et al., Astron. Astrophys. 580, L13 (2015)

D. Saumon et al., Astrophys. J. 460, 993 (1996)

H.E. Schlichting, Astrophys. J. Lett. 795, L15 (2014)

H.E. Schlichting et al., Icarus 247, 81 (2015)

A. Seiff et al., Science 272, 844 (1996)

A.P. Showman, T. Guillot, Astron. Astrophys. 385, 166 (2002)

C. Spalding, K. Batygin, Astrophys. J. 811, 82 (2015)

D.S. Spiegel, A.S. Burrows, Astrophys. J. 745, 174 (2012)

D.S. Spiegel et al., Astrophys. J. 727, 57 (2011)

S.W. Stahler, F. Palla, The Formation of Stars (2005)

D. Stamatellos, Astrophys. J. Lett. 810, L11 (2015)

D. Stamatellos, A.P. Whitworth, Mon. Not. R. Astron. Soc. 392(1), 413 (2009)

J.H. Steffen, Mon. Not. R. Astron. Soc. 433, 3246 (2013)

D.J. Stevenson, Annu. Rev. Earth Planet. Sci. 10, 257 (1982)

D.J. Stevenson, Icarus 62, 4 (1985)

D.J. Stevenson, E.E. Salpeter, Astrophys. J. Suppl. Ser. 35, 221 (1977)

J. Szulágyi et al., Astrophys. J. 782, 65 (2014) 
H. Tanaka et al., Astrophys. J. 565, 1257 (2002)

T. Tanigawa et al., Astrophys. J. 747, 47 (2012)

C.E.J.M.L.J. Terquem, Mon. Not. R. Astron. Soc. 341, 1157 (2003)

L. Testi et al., Astron. Astrophys. 403, 323 (2003)

A. Toomre, The structure and evolution of normal galaxies, in Proceedings of the Advanced Study Institute (1981), p. 111

A.H.M.J. Triaud et al., Astron. Astrophys. 524, A25 (2010)

S. Udry, M. Mayor, in Extreme Solar Systems, ed. by D. Fischer, F.A. Rasio, S.E. Thorsett, A. Wolszczan. Astronomical Society of the Pacific Conference Series, vol. 398 (2008), p. 13

H.C. Urey, Proc. Natl. Acad. Sci. 41, 127 (1955)

A. Uribe et al., Astrophys. J. 802, 54 (2015)

N. Vaytet et al., Astron. Astrophys. 557, A90 (2013)

A. Vidal-Madjar et al., Astrophys. J. Lett. 676, L57 (2008)

W.R. Ward, Icarus 126, 261 (1997)

G.W. Wetherill, G.R. Stewart, Icarus 77, 330 (1989)

D.J. Wilner et al., Astrophys. J. Lett. 626, L109 (2005)

H.F. Wilson, B. Militzer, Astrophys. J. 745, 54 (2012)

J.N. Winn, D.C. Fabrycky, Annu. Rev. Astron. Astrophys. 53, 409 (2015)

J.N. Winn et al., Astrophys. J. 631, 1215 (2005)

Y. Wu, N. Murray, Astrophys. J. 589, 605 (2003)

A.N. Youdin, Y. Lithwick, Icarus 192, 588 (2007)

J.-P. Zahn, Astron. Astrophys. 57, 383 (1977)

Z. Zhu, Astrophys. J. 799, 16 (2015)

Z. Zhu et al., Astrophys. J. 729, 47 (2011)

Z. Zhu et al., Astrophys. J. 755, 6 (2012)

Z. Zhu et al., Astrophys. J. 768, 143 (2013)

W. Zhu et al., Astrophys. J. 796(1), 67 (2014)

Z. Zhu et al., Astrophys. J. 813, 88 (2015) 